REPRESENTATION THEORY

An Electronic Journal of the American Mathematical Society

Volume 3, Pages 32-57 (June 2, 1999)

S $1088-4165(99) 00045-X$

\title{
QUATERNIONIC DISCRETE SERIES
}

\author{
DEREK GORDON
}

ABstract. This work investigates the discrete series of linear connected semisimple noncompact groups $G$. These are irreducible unitary representations that occur as direct summands of $L^{2}(G)$.

Harish-Chandra produced discrete series representations, now called holomorphic discrete series representations, for groups $G$ with the property that, if $K$ is a maximal compact subgroup, then $G / K$ has a complex structure such that $G$ acts holomorphically. Holomorphic discrete series are extraordinarily explicit, it being possible to determine all the elements in the space and the action by the Lie algebra of $G$.

Later Harish-Chandra parametrized the discrete series in general. His argument did not give an actual realization of the representations, but later authors found realizations in spaces defined by homology or cohomology. These realizations have the property that it is not apparent what elements are in the space and what the action of the Lie algebra $G$ is.

The point of this work is to find some intermediate ground between the holomorphic discrete series and the general discrete series, so that the intermediate cases may be used to get nontrivial insights into the internal structure of the discrete series in the general case.

The author examines the Vogan-Zuckerman realization of discrete series by means of cohomological induction. An explicit complex for computing the homology on the level of a $K$ module was already known. Also, Duflo and Vergne had given information about how to compute the action of the Lie algebra of $G$.

The holomorphic discrete series are exactly those cases where the representations can be realized in homology of degree 0 . The intermediate cases that are studied are those where the representation can be realized in homology of degree 1. Many of the intermediate cases correspond to the situation where $G / K$ has a quaternionic structure. The author obtains general results for $\mathrm{A}_{\mathfrak{q}}(\lambda)$ discrete series in the intermediate case.

\section{Contents}

Introduction 33

1. Construction of discrete series by cohomological induction 34

1.1. Discrete series $\quad 34$

1.2. Cohomological induction 37

1.3. Useful theorems 43

2. $\mathfrak{g}$ action for $\mathrm{A}_{\mathfrak{q}}(\lambda)$ minimal $K$ type $\quad 45$

Acknowledgements $\quad 55$

$\begin{array}{ll}\text { References } & 56\end{array}$

Received by the editors February 19, 1998 and, in revised form, November 18, 1998. 1991 Mathematics Subject Classification. Primary 22E46.

(c)1999 American Mathematical Society 


\section{INTRODUCTION}

This paper investigates the discrete series of linear connected semisimple noncompact groups $G$. These are irreducible unitary representations that occur as direct summands of $L^{2}(G)$.

It was Bargmann [Bar] who discovered that such representations can actually exist. In classifying the irreducible unitary representations for $G=S L(2, \mathbb{R})$, he found two countable families of discrete series, one in spaces of analytic functions on the unit disk and one in the space of complex conjugates.

Later Harish-Chandra ([HC1], [HC2] and [HC3]) abstracted Bargmann's construction and generalized it somewhat. Let $K$ be a maximal compact subgroup of $G$. The setting for Harish-Chandra's generalization is that $G / K$ has a complex structure such that $G$ acts holomorphically. For each irreducible finite-dimensional representation of $K$ whose highest weight satisfies a certain negativity property, Harish-Chandra considered the space of scalar-valued square-integrable holomorphic functions on $G$ that transform under a maximal torus of $K$ by the given highest weight, with $G$ acting by translation on the functions. Harish-Chandra found that this space gave a discrete series representation, and such representations have come to be known as holomorphic discrete series. Holomorphic discrete series are extraordinarily explicit, and one can read off with relative ease what elements are in the space and how the Lie algebra of $G$ operates.

Harish-Chandra's early work on the Plancherel formula for semisimple groups suggested that other groups should have discrete series, not just those with $G / K$ complex, and yet no such representations were discovered for a number of years. Then in 1960 Dixmier [Dix] was able to classify the irreducible unitary representations of $G=S O(4,1)$, as well as its double cover, and to prove that some of his representations were discrete series. Dixmier's student Takahashi [Tak] gave global realizations of these representations, ostensibly explicit, and for the first time one had nonholomorphic examples.

In 1966 Harish-Chandra [HC4] succeeded in parametrizing all the discrete series for all semisimple groups $G$ for which the rank of $G$ equals the rank of the maximal compact $K$, and he showed that there were no discrete series if the equal rank condition failed. His parametrization was in terms of features of the global characters of such representations and did not give a global realization of any kind, other than as an unspecified subspace of $L^{2}(G)$.

Global realizations were the subject of the Langlands conjecture [Lan], which ultimately was proved by Schmid ([S1], [S2] and [S3]). It was shown that the discrete series can be realized as spaces of $L^{2}$ cohomology sections over $G / T$, where $T$ is a maximal torus of $G$. Although the result was a space that one could define, neither the methods of proof nor direct computations showed how to produce a single nonzero element in the representation space.

Thus, except for $S O(4,1)$ and some similar examples that were considered afterward, there was no intermediate ground between the very concrete holomorphic discrete series and the very abstract general discrete series. In fact, closer examination of Takahashi's construction shows that it is less explicit than one might at first suppose. It does allow for the computation of nonzero elements in discrete series, one representation at a time, but it does not really show what the whole space is like nor what the action of the Lie algebra of $G$ is. 
The point of this paper is to find some intermediate ground between the holomorphic discrete series and the general discrete series, with the hope that one can use the intermediate cases to get nontrivial insights into the internal structure of the representations in the general case.

Thus we seek a kind of discrete series, more complicated than the holomorphic type, for which a global realization is relatively concrete. We insist on being able to identify the elements in the space, and we want to understand the action of the Lie algebra of $G$ rather explicitly.

By now there are several "constructions" of general discrete series. In addition to Schmid's, we mention the Hotta-Parthasarathy realization [H-R] using a Dirac operator, the Enright-Varadarajan realization [E-V] using an iterative cohomological construction, the Flensted-Jensen realization ([FJ1] and [FJ2]) using a kind of spherical function for a dual group, and the Vogan-Zuckerman realization ([Vog] and $[\mathrm{Zuc}])$ using cohomological induction.

Our approach to the problem is to find one of these general constructions that is relatively manageable in some situations. The general construction that we examine is the Vogan-Zuckerman realization using cohomological induction. This particular construction seems favorable since the homology or cohomology is to be computed on the level of a $K$ module from an explicit complex and since Duflo and Vergne $[\mathrm{D}-\mathrm{V}]$ have given information about how to compute the action by the Lie algebra of $G$. All the cases of holomorphic discrete series, and only those, turn out to be realizable in homology or cohomology of degree 0 , and we propose cases where the realization is in degree 0 or 1 as the intermediate case.

Interestingly, Wolf [Wol] has classified those groups for which $G / K$ has a reasonable quaternion structure, and it turns out that most of the situations where Vogan-Zuckerman representations are realized in degree 1 are of the kind on Wolf's list. The study of discrete series that arise from Wolf's situation is not new but has been considered by Enright, Parthasarathy, Wallach, and Wolf ([EW1] and [EW2]), although these authors had completely different objectives.

To summarize our results, in Chapter 1, the Vogan-Zuckerman construction of discrete series by means of cohomological induction is described. Crucial items are the $K$ modules (1.12) and the maps $\partial_{n}$ and $\partial_{n}^{\natural}$ (1.16) and (1.17b) acting on these modules. In Chapter 2, we consider the discrete series $\mathrm{A}_{\mathfrak{q}}(\lambda)$ for any group $G$ satisfying the conditions of Theorem 1.6(a). The first main result is Proposition 2.3 , which states explicitly a basis vector for the multiplicity space of the minimal $K$ type. Other results include Proposition 2.5, which gives a general form for any $K$ type appearing in $\mathrm{A}_{\mathfrak{q}}(\lambda)$. A consequence of the proof of Proposition 2.6 is the relation $(2.6 \mathrm{~g})$, which gives a general form for any $K$ type $\eta$ that has $\left.\mathcal{C}_{2}^{*}\left(\mathbb{C}_{\lambda}\right)\right|_{\eta}$ nonzero $\left(\left.\mathcal{C}_{2}^{*}\left(\mathbb{C}_{\lambda}\right)\right|_{\eta}\right.$ defined in Definition 1.19). Theorem 2.7 is our main result. It shows that computation of the action of the Lie algebra of $G$ on a homology basis for the minimal $K$ type is a solvable problem. Finally, Proposition 2.8 proves that there are $K$ types $\eta$ for which $\left.\mathcal{C}_{2}^{*}\left(\mathbb{C}_{\lambda}\right)\right|_{\eta}$ is nonzero. This result is important when determining a homology basis.

\section{Construction of Discrete Series by COHOMOlOGical induCtion}

1.1. Discrete series. For a unimodular group $G$, an irreducible unitary representation $\pi$ is in the discrete series if it is a direct summand of the right regular 
representation on $L^{2}(G)$, or equivalently if some (or equivalently every) nonzero matrix coefficient $\left(\pi(g) v_{1}, v_{2}\right)$ is in $L^{2}(G)[\mathrm{K}-\mathrm{V}, \mathrm{p} .20]$.

Let $G$ be a linear connected semisimple noncompact Lie group, and let $K$ be a maximal compact subgroup. The discrete series representations for $G$ were parametrized by Harish-Chandra [HC4]. A key result is that the discrete series representations exist for $G$ if and only if a maximal torus $T$ of $K$ is maximally abelian in $G$. This is equivalent to saying rank $G=\operatorname{rank} K$. For an exposition, see [K3, p. 454], especially Theorem 9.20.

The Vogan-Zuckerman construction known as cohomological induction is a way of constructing discrete series. At this point we shall give a brief sketch of cohomological induction, omitting details of a number of definitions. In the course of our description, we shall note under what conditions the construction yields discrete series. We shall give a more detailed description of cohomological induction in the next section. Motivation for cohomological induction may be found in $[\mathrm{K}-\mathrm{V}$, Introduction], particularly sections 3 and 5, or [K1, Section 1(Setting)].

We begin with $G$ and $K$ as stated above. For $T$ a torus subgroup of $K$ (not necessarily maximally abelian), we let $L=Z_{G}(T)$ be the centralizer of $T$ in $G$. Eventually we shall assume that $L \subseteq K$, but we do not make this assumption yet. Let $\mathfrak{g}_{0}$ be the Lie algebra of $G, \mathfrak{g}=\left(\mathfrak{g}_{0}\right)^{\mathbb{C}}$, let $\mathfrak{k}$ and $\mathfrak{l}$ be the complexifications of the Lie algebras of $K$ and $L$, respectively, and let $\mathfrak{q}$ be a $\theta$-stable parabolic subalgebra of $\mathfrak{g}$ of the form $\mathfrak{q}=\mathfrak{l} \oplus \mathfrak{u}$, where $\mathfrak{u}$ is the nilpotent radical. Here and elsewhere, we shall use the notation $\mathfrak{m}_{0}$, with subscript 0 , to refer to a real Lie algebra, and we shall use the notation $\mathfrak{m}$ without subscript to refer to a complexified Lie algebra, i.e., $\mathfrak{m}=\left(\mathfrak{m}_{0}\right)^{\mathbb{C}}$. Define $\overline{\mathfrak{q}}=\mathfrak{l} \oplus \overline{\mathfrak{u}}$, where bar denotes conjugation of $\mathfrak{g}$ with respect to $\mathfrak{g}_{0}$. Then $\mathfrak{g}=\mathfrak{u} \oplus \mathfrak{l} \oplus \overline{\mathfrak{u}}$.

What follows mirrors closely the discussion in [K-V, pp. 26-27]. Suppose $Z$ is an irreducible $(\mathfrak{l}, L \cap K)$ module. We define $Z^{\#}$, an $(\mathfrak{l}, L \cap K)$ module, by

$$
Z^{\#}=Z \otimes \bigwedge_{\text {top }} \mathfrak{u}
$$

where top $=\operatorname{dim}(\mathfrak{u})$. Here and elsewhere we use the notation $\otimes$ with no subscript to mean $\otimes_{\mathbb{C}}$, the tensor product over $\mathbb{C}$.

We regard $Z^{\#}$ as a $(\overline{\mathfrak{q}}, L \cap K)$ module on which $\overline{\mathfrak{u}}$ acts as 0 . The next step is to apply an 'algebraic induction' functor to form a $(\mathfrak{g}, L \cap K)$ module

$$
\operatorname{ind}_{\mathfrak{q}, L \cap K}^{\mathfrak{g}, L \cap K}\left(Z^{\#}\right)=U(\mathfrak{g}) \otimes_{\overline{\mathfrak{q}}} Z^{\#} \cong U(\mathfrak{u}) \otimes Z^{\#} .
$$

After this we apply the $j$ th derived functor of the "Bernstein functor" $\Pi_{\mathfrak{g}, L \cap K}^{\mathfrak{g}, K}$ to obtain a $(\mathfrak{g}, K)$ module:

$$
\mathcal{L}_{j}(Z)=\left(\Pi_{\mathfrak{g}, L \cap K}^{\mathfrak{g}, K}\right)_{j}\left(\operatorname{ind}_{\mathfrak{q}, L \cap K}^{\mathfrak{g}, L \cap K}\left(Z^{\#}\right)\right) .
$$

As mentioned above, under certain conditions, namely for the particular value $j=S=\operatorname{dim}(\mathfrak{u} \cap \mathfrak{k})$ and for certain irreducible modules $Z$, the $(\mathfrak{g}, K)$ module $\mathcal{L}_{j}(Z)$ is a discrete series representation. We will state these conditions on $Z$ presently, but first we finish discussing how discrete series are constructed using $\mathcal{L}_{j}(Z)$. Under the assumption that the conditions for $j$ and $Z$ are satisfied, $\mathcal{L}_{j}(Z)$ can be computed directly by means of a complex. See formula (1.12) of the next section for details. Although $\mathcal{L}_{j}(Z)$ is a $(\mathfrak{g}, K)$ module, the complex whose members come from (1.12) will provide us with a $(\mathfrak{k}, K)$ module. This $(\mathfrak{k}, K)$ module is the $S$ th homology of the complex, and we can reimpose the full $\mathfrak{g}$ action on this $(\mathfrak{k}, K)$ module by using a 
theorem of Duflo and Vergne [K-V, Proposition 3.80] in combination with another result [K-V, Proposition 3.83]. To be consist with our numbering, we refer to these as Propositions 1.20 and 1.21, respectively. They may be found in Section 1.3 of this chapter.

At this point, we describe the the conditions on the irreducible $(\mathfrak{l}, L \cap K)$ module $Z$ in order that $\mathcal{L}_{S}(Z)$ be a discrete series. The first condition is that $L$ be compact, so that $L \subseteq K$. Under this condition, let us fix a maximal torus $B$ in $L$. $B$ will be a maximal torus also in $K$, and we let $\mathfrak{b}$ be the complexified Lie algebra. Form the roots $\Delta=\Delta(\mathfrak{g}, \mathfrak{b})$, and choose a positive system $\Delta^{+}$compatible with $\mathfrak{q}$. If $Z$ is an irreducible $(\mathfrak{l}, L)$ module with highest weight $\lambda$, then the infinitesimal character of $\mathcal{L}_{S}(Z)$ is $\lambda+\delta(\mathfrak{g})$, where $\delta(\mathfrak{g})$ is one half the sum of the positive roots of $\mathfrak{g}$. The necessary condition for $\mathcal{L}_{S}(Z)$ to be a discrete series is that $\lambda+\delta(\mathfrak{g})$ be dominant and nonsingular for $\mathfrak{g}$. In other words,

$$
\langle\lambda+\delta(\mathfrak{g}), \alpha\rangle>0 \quad \text { for all } \alpha \in \Delta^{+} .
$$

In this case $\mathcal{L}_{S}(Z)$ is the discrete series with Harish-Chandra parameter $\lambda+\delta(\mathfrak{g})$, in the sense of [HC4]. It turns out that $\delta(\mathfrak{g})=\delta(\mathfrak{l})+\delta(\mathfrak{u})$, and because $L$ is compact for our cases of interest, then $\lambda+\delta(\mathfrak{l})$ is the infinitesimal character of $Z$. We have arranged that $\Delta(\mathfrak{u})$, the roots of $\mathfrak{u}$, are all positive, and we have seen that $\delta(\mathfrak{u}) \perp \Delta(\mathfrak{l})$. Because $\lambda+\delta(\mathfrak{l})$ is strictly dominant with respect to the roots of $\mathfrak{l}$, we see that $\lambda+\delta(\mathfrak{g})$ is automatically strictly dominant with respect to $\Delta^{+}(\mathfrak{l})$. Therefore, the inequality (1.4a) can be rewritten as

$$
\langle(\text { infinitesimal character of } Z)+\delta(\mathfrak{u}), \alpha\rangle>0 \quad \text { for all } \alpha \in \Delta(\mathfrak{u}) \text {. }
$$

We mentioned in the introduction that the value $S=\operatorname{dim}(\mathfrak{u} \cap \mathfrak{k})$ may be of interest in singling out special families of discrete series representations. We therefore state theorems that classify the groups $G$ and parabolic subalgebras $\mathfrak{q}$ corresponding to the cases $S=0$ and $S=1$. The proofs may be found in Gordon's dissertation [Gor].

Theorem 1.5. Let $G$ be a noncompact simple group with rank $G=\operatorname{rank} K$, and suppose that the $\theta$ stable parabolic subalgebra $\mathfrak{q}=\mathfrak{l} \oplus \mathfrak{u}$ of $\mathfrak{g}$ has $S=\operatorname{dim}(\mathfrak{u} \cap \mathfrak{k})$ equal to 0 . Then either $\mathfrak{q}=\mathfrak{g}$ or else $G / K$ is Hermitian symmetric with $\mathfrak{l}=\mathfrak{k}$ and $\mathfrak{u}=\mathfrak{p}^{+}$in a suitable good ordering on the roots.

Theorem 1.6. Let $G$ be a noncompact simple group with rank $G=\operatorname{rank} K$, and suppose that the $\theta$ stable parabolic subalgebra $\mathfrak{q}=\mathfrak{l} \oplus \mathfrak{u}$ of $\mathfrak{g}$ has $S=\operatorname{dim}(\mathfrak{u} \cap \mathfrak{k})$ equal to 1 . Let $\beta$ be the unique positive compact root in $\Delta(\mathfrak{u})$. Then $\{\beta,-\beta\}$ is a simple component in the root system of $\mathfrak{k}$, and the following is a classification of the possibilities for the Dynkin diagram of $\mathfrak{g}$ and the roles of $\mathfrak{l}$ and $\mathfrak{u}$ :

(a) $\Delta(\mathfrak{u})$ contains exactly one simple root $\beta_{0}$ of $\mathfrak{g}, \beta_{0}$ is noncompact, all roots of $\mathfrak{l}$ are compact, $\beta$ is the largest root and contains $\beta_{0}$ in its simple-root expansion with coefficient 2 , and $\beta_{0}$ is characterized as the unique simple root nonorthogonal to $\beta$;

(b) $\Delta(\mathfrak{u})$ contains exactly one simple root $\beta_{0}$ of $\mathfrak{g}, \beta_{0}$ is a node in a Dynkin diagram of type $\mathrm{A}_{n}$ with $n \geq 2, \beta_{0}$ is noncompact, exactly two other simple roots are noncompact and they are adjacent, and $\beta$ is the sum of the simple roots from $\beta_{0}$ through the nearer noncompact simple root of $\mathfrak{l}$;

(c) $\Delta(\mathfrak{u})$ contains exactly one simple root $\beta_{0}$ of $\mathfrak{g}, \beta_{0}$ is a node in a Dynkin diagram of type $\mathrm{A}_{n}$ with $n \geq 2, \beta_{0}$ is noncompact, exactly one other simple 
root is noncompact and it is the other node, and $\beta$ is the sum of all the simple roots;

(d) $\Delta(\mathfrak{u})$ contains exactly one simple root $\beta_{0}$ of $\mathfrak{g}, \beta_{0}$ is a node in a Dynkin diagram of type $\mathrm{A}_{n}$ with $n \geq 2, \beta_{0}$ is compact, the simple root adjacent to $\beta_{0}$ is the one and only noncompact simple root, and $\beta$ equals $\beta_{0}$;

(e) $\Delta(\mathfrak{u})$ contains exactly one simple root $\beta_{0}$ of $\mathfrak{g}, \mathfrak{g}_{0}$ is $\mathfrak{s p}(2, \mathbb{R})$ of type $\mathrm{C}_{2}, \beta_{0}$ is compact and short, the other simple root is noncompact and long, and $\beta$ equals $\beta_{0}$;

(f) $\Delta(\mathfrak{u})$ contains exactly one simple root $\beta_{0}$ of $\mathfrak{g}, \mathfrak{g}_{0}$ is split $\mathrm{G}_{2}, \beta_{0}$ is long and noncompact, the other simple root is short and compact, $\beta$ contains $\beta_{0}$ in its simple-root expansion with coefficient 2 , and $\beta$ is the largest short root;

(g) $\Delta(\mathfrak{u})$ contains exactly two simple roots $\beta_{1}$ and $\beta_{2}$, the Dynkin diagram of $\mathfrak{g}$ is of type $\mathrm{A}_{n}$ with $n \geq 2, \beta_{1}$ and $\beta_{2}$ are the nodes, $\beta_{1}$ and $\beta_{2}$ are noncompact, all other simple roots are compact, and $\beta$ is the sum of all the simple roots;

(h) $\Delta(\mathfrak{u})$ contains exactly two simple roots $\beta_{1}$ and $\beta_{2}, \beta_{1}$ is noncompact and $\beta_{2}$ is compact, the Dynkin diagram of $\mathfrak{g}$ is of type $\mathrm{A}_{n}, \beta_{2}$ is a node, $\beta_{1}$ is adjacent to $\beta_{2}$, the simple roots of $\mathfrak{l}$ are all compact, and $\beta$ is $\beta_{2}$;

(i) $\Delta(\mathfrak{u})$ contains exactly two simple roots $\beta_{1}$ and $\beta_{2}$, $\mathfrak{g}_{0}$ is $\mathfrak{s p}(2, \mathbb{R})$ of type $\mathrm{C}_{2}$, at least one of $\beta_{1}$ and $\beta_{2}$ is noncompact, and $\beta$ is the unique positive compact root.

The situation described in Theorem 1.5 where $S=0$ leads exactly to holomorphic discrete series, but, in reality, as vector-valued holomorphic functions on $G / K$. Alternatively, these representations are being presented on the level of $(\mathfrak{g}, K)$ modules as generalized Verma modules, which are reasonably well understood. Our approach toward finding intermediate cases of discrete series using cohomological induction will be to look at situations where $S=1$. The main case of Theorem 1.6 is (a), the other cases correspond to particular groups. Case (a) is closely related to the work of Wolf [Wol] on quaternion structure for $G / K$. See also [Sud], [Bes], $[\mathrm{F} 1],[\mathrm{F} 2]$ and [F3]. The term quaternionic discrete series will be used to refer to discrete series representations of a group $G$ whose complexified Lie algebra $\mathfrak{g}$ satisfies the conditions of case (a) in Theorem 1.6.

Section 1.2 of this chapter gives a more detailed description of cohomological induction, providing definitions and giving concrete formulas for the complexes used in determining discrete series representations. Section 1.3 presents theorems that will be used in Chapter 2 .

In order to define the term $K$ type, we need some theory. The Peter-Weyl Theorem [K3, Theorem 1.12] states that $\mathcal{L}_{S}(Z)$, when thought of as a $K$ representation, will decompose as the direct sum of irreducible, finite-dimensional $K$ representations. Each of these irreducible representations is parametrized, up to unitary equivalence, by its highest weight (Theorem of the Highest Weight, [K3, Theorem 4.28]). The highest weights that appear with nonzero multiplicity in such a direct sum decomposition are called the $K$ types of $\mathcal{L}_{S}(Z)$.

1.2. Cohomological induction. We begin this section by defining some terms used in our discussion of cohomological induction. After these definitions are stated, we present a more thorough analysis of the objects and maps used in constructing discrete series representations by means of cohomological induction. 
Definition 1.7. We define the Hecke Algebra $R(K)$ as the direct sum

$$
R(K)=\bigoplus_{\mu \in \hat{K}} V_{\mu} \otimes\left(V_{\mu}\right)^{*}
$$

where $\hat{K}$ is the set of equivalence classes of irreducible representations of $K,\left(\pi, V_{\mu}\right)$ is an irreducible representation of $K$ of highest weight $\mu$ and $\left(\pi^{*},\left(V_{\mu}\right)^{*}\right)$ is the contragredient representation. A more detailed definition is given in $[\mathrm{K}-\mathrm{V},(1.33)]$. From this reference we see that both $l=$ left regular representation and $r=$ right regular representation are defined on $R(K)$. We shall use the following fact repeatedly throughout this paper: For any pure tensor $v \otimes v^{*}$ in a summand $V_{\mu} \otimes\left(V_{\mu}\right)^{*}$ of $R(K)$,

$$
\begin{aligned}
& l(k)\left(v \otimes v^{*}\right)=(\pi(k) v) \otimes v^{*}, \\
& r(k)\left(v \otimes v^{*}\right)=v \otimes\left(\pi^{*}(k) v^{*}\right) .
\end{aligned}
$$

This extends to linear combinations of pure tensors, as well as finite sums of the summands $V_{\mu} \otimes\left(V_{\mu}\right)^{*}$. A thorough discussion of $R(K)$ can be found in [K-V, Chapter 1]. The summands $V_{\mu} \otimes\left(V_{\mu}\right)^{*}$ of $R(K)$ are called the $K$ isotypic components of $R(K)$ of type $\mu$. See [K-V, Proposition 1.18] for a further discussion.

Definition 1.8. We define the Hecke Algebra $R(\mathfrak{g}, K)$ to be the tensor product

$$
R(K) \otimes_{U(\mathfrak{k})} U(\mathfrak{g}),
$$

where $U(\mathfrak{k})$ is the universal enveloping algebra of $\mathfrak{k}$, contained in $U(\mathfrak{g})$, the universal enveloping algebra of $\mathfrak{g}$. Technically speaking, this tensor product is isomorphic to the actual Hecke Algebra $R(\mathfrak{g}, K)[\mathrm{K}-\mathrm{V}$, Corollary 1.71], but for our purposes the tensor product is sufficient. Note that $R(K)$ is a right $U(\mathfrak{k})$ module via the action

$$
T v=r\left(v^{t}\right) T,
$$

where $T \in R(K)$ and $v \in U(\mathfrak{k})$. The transpose map $v \mapsto v^{t}$ is an anti-automorphism of $U(\mathfrak{k})$ characterized by

$$
X^{t}=-X \quad \text { and } \quad(u v)^{t}=v^{t} u^{t}
$$

for $X \in \mathfrak{k}$ and $u, v \in U(\mathfrak{k})$. In (1.8a), $r$ is right regular representation extended to $U(\mathfrak{k})$. Note also that $U(\mathfrak{g})$ is a left $U(\mathfrak{k})$ module in a natural way, namely by left multiplication. A thorough discussion of $R(\mathfrak{g}, K)$ is given in [K-V, Chapter 1]. In the first section of that chapter is a discussion of the transpose map and the extension of $r$ to the universal enveloping algebra.

Definition 1.9. Suppose that $i:(\mathfrak{h}, L) \rightarrow(\mathfrak{g}, K)$ is a map of pairs $[\mathrm{K}-\mathrm{V},(2.6)]$, i.e., a pair of maps

$$
\begin{array}{ll}
i_{\text {alg }}: \mathfrak{h} \rightarrow \mathfrak{g}, & \text { a Lie algebra homomorphism, } \\
i_{\mathrm{gp}}: L \rightarrow K, & \text { a Lie group homomorphism }
\end{array}
$$

satisfying the compatibility conditions

(i) $i_{\text {alg }} \circ \iota_{L}=\iota_{K} \circ d i_{\mathrm{gp}}$, where $d i_{\mathrm{gp}}$ is the differential of $i_{\mathrm{gp}}$;

(ii) $i_{\text {alg }} \circ \operatorname{Ad}_{L}(l)=\operatorname{Ad}_{K}\left(i_{\mathrm{gp}}(l)\right) \circ i_{\text {alg }}$ for $l \in L$.

For $V$ an approximately unital $(\mathfrak{h}, L)$ module, the functor $P(\cdot)$ is defined by

$$
P(V)=P_{\mathfrak{h}, L}^{\mathfrak{g}, K}(V)=R(\mathfrak{g}, K) \otimes_{R(\mathfrak{h}, L)} V
$$


This is a $(\mathfrak{g}, K)$ module. A special example of the $P$ functor occurs when $\mathfrak{h}=\mathfrak{g}$. For $V$ an approximately unital $(\mathfrak{g}, L)$ module, the Bernstein functor $P_{\mathfrak{g}, L}^{\mathfrak{g}, K}$, denoted by $\Pi_{\mathfrak{g}, L}^{\mathfrak{g}, K}$, satisfies the $K$ isomorphism

$$
P_{\mathfrak{g}, L}^{\mathfrak{g}, K}(V)=\Pi_{\mathfrak{g}, L}^{\mathfrak{g}, K}(V)=R(K) \otimes_{R(\mathfrak{k}, L)} V,
$$

which is proved in Proposition 2.69 of $[\mathrm{K}-\mathrm{V}]$. Another example of the $P$ functor occurs when $L=K$. For $V$ an approximately unital $(\mathfrak{h}, L)$ module, the functor $P_{\mathfrak{h}, L}^{\mathfrak{g}, L}$, called Lie algebra induction, satisfies the equality

$$
P_{\mathfrak{h}, L}^{\mathfrak{g}, L}(V) \cong \operatorname{ind}_{\mathfrak{h}, L}^{\mathfrak{g}, L}(V)=U(\mathfrak{g}) \otimes_{U(\mathfrak{h})} V,
$$

with $L$ acting by the tensor product of Ad and the action on $V$. This equality is proved in Proposition 2.57 of $[\mathrm{K}-\mathrm{V}]$.

The previous definition was given for a general map of pairs. In what follows, we specialize to the case of quaternionic discrete series, so that $\mathfrak{q}$ is a parabolic subalgebra of $\mathfrak{g}, L \subseteq K$ is compact, $\mathfrak{u}$ is the nilradical, $\mathfrak{q}=\mathfrak{l} \oplus \mathfrak{u}$, and $\overline{\mathfrak{q}}=\mathfrak{l} \oplus \overline{\mathfrak{u}}$.

Definition 1.10. The functor $\mathcal{F}(\cdot)$, the forgetful functor, will arise in two ways in this paper. For the first case, suppose $V$ an approximately unital $(\mathfrak{l}, L)$ module, $\mathcal{F}_{\mathfrak{l}, L}^{\bar{q}, L}(V)$ is the $(\mathfrak{l}, L)$ module $V$, extended to be a $(\overline{\mathfrak{q}}, L)$ module by defining $X v=0$ for $X \in \overline{\mathfrak{u}}$ and $v \in V$.

In the second case, $\mathfrak{g}=\mathfrak{k} \oplus \mathfrak{p}$ is the Cartan decomposition of $\mathfrak{g}$. The functor $\mathcal{F}_{\mathfrak{g}, L}^{\mathfrak{k}, L}(V)$ is the $(\mathfrak{g}, L)$ module $V$, reduced to a $(\mathfrak{k}, L)$ module by ignoring the action of $\mathfrak{p}$ on $V$. A precise definition of the functor $\mathcal{F}$ can be found in [K-V, p. 109].

We now state precisely what complexes are used in determining $\mathcal{L}_{S}(Z)$. For this discussion we will continue with the notation for groups and Lie algebras stated in Section 1.1 of this chapter. We will assume that appropriate choices have been made for $S$ and $Z$ so that $\mathcal{L}_{S}(Z)$ is a discrete series representation (see Section 1.1). If we abbreviate ind $\operatorname{iq}_{\mathfrak{q}, L}^{\mathfrak{g}, L}\left(Z^{\#}\right)$ by $V_{Z}$, then the precise complex used consists of the $(\mathfrak{g}, K)$ modules

$$
R(\mathfrak{g}, K) \otimes_{L}\left(\bigwedge^{n}(\mathfrak{u} \oplus \overline{\mathfrak{u}}) \otimes V_{Z}\right)
$$

and a map $\partial_{n}$, which maps from $\bigwedge^{n}$ to $\bigwedge^{n-1}$, defined on a module (1.11a) as

$$
\begin{aligned}
& \partial_{n}\left(R \otimes\left(Y_{1} \wedge \cdots \wedge Y_{n} \otimes v\right)\right) \\
& =\sum_{l=1}^{n}(-1)^{l+1}\left(R Y_{l} \otimes\left(Y_{1} \wedge \cdots \wedge \widehat{Y}_{l} \wedge \cdots \wedge Y_{n} \otimes v\right)\right) \\
& \quad+\sum_{l=1}^{n}(-1)^{l}\left(R \otimes\left(Y_{1} \wedge \cdots \wedge \widehat{Y}_{l} \wedge \cdots \wedge Y_{n} \otimes Y_{l} v\right)\right)
\end{aligned}
$$

for $R \in R(\mathfrak{g}, K), Y_{1}, \ldots, Y_{n} \in \mathfrak{u} \oplus \overline{\mathfrak{u}}$, and $v \in V_{Z}$. The value $n$ in (1.11a) ranges between 0 and $N=\operatorname{dim}(\mathfrak{g} / \mathfrak{l})$. This complex is discussed thoroughly in $[\mathrm{K}-\mathrm{V}$, Chapter 2, Section 7]. Formulas (1.11a) and (1.11b) above are taken from formulas (2.128a) and (2.128b) of this reference and modified to fit our situation. Using this notation, $\mathcal{L}_{S}(Z)$ is the $(\mathfrak{g}, K)$ module $\operatorname{Ker} \partial_{S} / \operatorname{Im} \partial_{S+1}$. 
Although we could calculate the discrete series in this way, we choose to modify this construction somewhat and produce the discrete series in steps. More specifically, the construction above gives us a $(\mathfrak{g}, K)$ module, which is what we desire ultimately. However, we proceed by first changing the complex (1.11a) to a (k, $K)$ module and then reconstructing the full $\mathfrak{g}$ action by means of Propositions 1.20 and 1.21 . The reason for this change is that our new complex replaces $R(\mathfrak{g}, K)$ with $R(K)$, which is much easier to manipulate. To make the change, we use Corollary 3.26(c) of $[\mathrm{K}-\mathrm{V}]$, which allows us to replace $\left(\Pi_{\mathfrak{g}, L}^{\mathfrak{g}, K}\right)_{S}\left(\operatorname{ind}_{\overline{\mathfrak{q}}, L}^{\mathfrak{g}, L} Z^{\#}\right)$ by $\left(\Pi_{\mathfrak{k}, L}^{\mathfrak{k}, K}\right)_{S}\left(\mathcal{F}_{\mathfrak{g}, L}^{\mathfrak{k}, L}\left(\operatorname{ind}_{\overline{\mathfrak{q}}, L}^{\mathfrak{g}, L} Z^{\#}\right)\right)$. If we abbreviate $\mathcal{F}_{\mathfrak{g}, L}^{\mathfrak{k}, L}\left(\operatorname{ind}_{\overline{\mathfrak{q}}, L}^{\mathfrak{g}, L} Z^{\#}\right)$ by $\mathcal{F}\left(V_{Z}\right)$, then (3.27) of the same reference tells us that the complex whose $S$ th homology is $\left(\Pi_{\mathfrak{k}, L}^{\mathfrak{k}, K}\right)_{S}\left(\mathcal{F}\left(V_{Z}\right)\right)$ is given by a complex whose modules are

$$
R(K) \otimes_{L}\left(\bigwedge^{n}((\mathfrak{u} \oplus \overline{\mathfrak{u}}) \cap \mathfrak{k}) \otimes V_{Z}\right)
$$

and whose maps $\partial_{n}$ are the same as that of (1.11b), except for notational changes. Note, however, that the domain of $n$ decreases when considering the $(\mathfrak{k}, K)$ modules (1.12) instead of the $(\mathfrak{g}, K)$ modules (1.11a). The module $\left(\Pi_{\mathfrak{k}, L}^{\mathfrak{k}, K}\right)_{S}\left(\mathcal{F}_{\mathfrak{g}, L}^{\mathfrak{k}, L}\left(\operatorname{ind}_{\overline{\mathfrak{q}}, L}^{\mathfrak{g}, L} Z^{\#}\right)\right)$, a $(\mathfrak{k}, K)$ module, is given by Ker $\partial_{S} / \operatorname{Im} \partial_{S+1}$, with $\partial_{S}$ and $\partial_{S+1}$ being maps on the modules (1.12).

A special kind of discrete series that we consider occurs when the $(\mathfrak{l}, L)$ module $Z$ mentioned before (1.1) is one-dimensional.

Definition 1.13. Let $B$ be a Cartan subgroup of $G$ with $\mathfrak{h}$ the complexified Lie algebra of $B$. Let $\lambda$ be an analytically integral linear functional on $\mathfrak{h}$ that is orthogonal to all the members of $\Delta(\mathfrak{l})$, and let $\mathbb{C}_{\lambda}$ be the corresponding $(\mathfrak{h}, B)$ module. By $[\mathrm{K}-\mathrm{V}$, Theorem 4.52], there is an irreducible $(\mathfrak{l}, L)$ module $Z$ with highest weight $\lambda$. Since $\lambda \perp \Delta(\mathfrak{l}), Z$ is one-dimensional, i.e., $Z=\mathbb{C}_{\lambda}$. Thus $Z$ becomes a onedimensional representation of $L$. We define a $(\mathfrak{g}, K)$ module by

$$
\mathrm{A}_{\mathfrak{q}}(\lambda)=\mathcal{L}_{S}\left(\mathbb{C}_{\lambda}\right)
$$

where, as before, $S=\operatorname{dim}(\mathfrak{u} \cap \mathfrak{k})$. For discussion see [K-V, Chapter 5, Example 2].

To make our calculations with $\partial_{n}$ simpler, we work with the space of $L$ invariants of

$$
R(K) \otimes \bigwedge^{n}((\mathfrak{u} \oplus \overline{\mathfrak{u}}) \cap \mathfrak{k}) \otimes V_{Z}
$$

denoted

$$
\left(R(K) \otimes \bigwedge^{n}((\mathfrak{u} \oplus \overline{\mathfrak{u}}) \cap \mathfrak{k}) \otimes V_{Z}\right)^{L}
$$

rather than with the space of $L$ coinvariants of (1.14a), namely (1.12). We can lift $\partial_{n}$ from (1.12) to (1.14a) where the formula for $\partial_{n}$ is still the same, but we no longer expect $\partial_{n}^{2}=0$. If $\xi$ denotes the representation of $L$ on $V_{Z}$, then $L$ acts on (1.14a) by $r \otimes \mathrm{Ad} \otimes \xi$ and we can recover (1.12), apart from a canonical isomorphism, as the subspace (1.14b) of (1.14a). The advantage is that we are now working with a subset of vectors rather than equivalence classes of vectors. For a fuller discussion, see $[\mathrm{K}-\mathrm{V}$, p. 193].

We simplify the process of determining elements in Ker $\partial_{n}$ for the (k, $\left.K\right)$ modules

$$
\left(V_{\mu} \otimes V_{\mu}^{*} \otimes \bigwedge^{n}((\mathfrak{u} \oplus \overline{\mathfrak{u}}) \cap \mathfrak{k}) \otimes V_{Z}\right)^{L}
$$


where $\mu$ is a $K$ type in the discrete series $\mathcal{L}_{S}(Z)$ and $V_{\mu} \otimes V_{\mu}{ }^{*}$ is the $K$ isotypic component of $R(K)$ of type $\mu$. We do this by first considering a pure tensor $v \otimes$ $v^{*} \otimes Y_{1} \wedge \cdots \wedge Y_{n} \otimes z$ in

$$
V_{\mu} \otimes V_{\mu}^{*} \otimes \bigwedge^{n}((\mathfrak{u} \oplus \overline{\mathfrak{u}}) \cap \mathfrak{k}) \otimes V_{Z} .
$$

Here $v \otimes v^{*} \in V_{\mu} \otimes V_{\mu}{ }^{*}$ and $z$ is a pure tensor in $V_{Z}$. From (1.11b) and (1.8a) we have

$$
\begin{aligned}
& \partial_{n}\left(v \otimes v^{*} \otimes Y_{1} \wedge \cdots \wedge Y_{n} \otimes z\right) \\
& =\sum_{l=1}^{n}(-1)^{l}\left(r\left(Y_{l}\right)\left(v \otimes v^{*}\right) \otimes Y_{1} \wedge \cdots \wedge \widehat{Y}_{l} \wedge \cdots \wedge Y_{n} \otimes z\right) \\
& \quad+\sum_{l=1}^{n}(-1)^{l}\left(v \otimes v^{*} \otimes Y_{1} \wedge \cdots \wedge \widehat{Y}_{l} \wedge \cdots \wedge Y_{n} \otimes Y_{l} z\right) \\
& =\sum_{l=1}^{n}(-1)^{l}\left(v \otimes \pi^{*}\left(Y_{l}\right) v^{*} \otimes Y_{1} \wedge \cdots \wedge \widehat{Y}_{l} \wedge \cdots \wedge Y_{n} \otimes z\right) \\
& \quad+\sum_{l=1}^{n}(-1)^{l}\left(v \otimes v^{*} \otimes Y_{1} \wedge \cdots \wedge \widehat{Y}_{l} \wedge \cdots \wedge Y_{n} \otimes Y_{l} z\right) \quad \text { from }(1.7 \mathrm{a}) \\
& =\sum_{l=1}^{n} v \otimes\left[(-1)^{l}\left(\pi^{*}\left(Y_{l}\right) v^{*} \otimes Y_{1} \wedge \cdots \wedge \widehat{Y}_{l} \wedge \cdots \wedge Y_{n} \otimes z\right)\right. \\
& \left.\quad+(-1)^{l}\left(v^{*} \otimes Y_{1} \wedge \cdots \wedge \widehat{Y}_{l} \wedge \cdots \wedge Y_{n} \otimes Y_{l} z\right)\right] \\
& =v \otimes \sum_{l=1}^{n}\left[(-1)^{l}\left(\pi^{*}\left(Y_{l}\right)^{*} \otimes Y_{1} \wedge \cdots \wedge \widehat{Y}_{l} \wedge \cdots \wedge Y_{n} \otimes z\right)\right. \\
& \left.\quad+(-1)^{l}\left(v^{*} \otimes Y_{1} \wedge \cdots \wedge \widehat{Y}_{l} \wedge \cdots \wedge Y_{n} \otimes Y_{l} z\right)\right] .
\end{aligned}
$$

Here, $r\left(Y_{l}\right)$ and $\pi^{*}\left(Y_{l}\right)$ refer to the differentials of the representations $r$ and $\pi^{*}$, both of which are defined on the group $K$. Here and elsewhere, we omit writing $\pi^{*}$ and simply write the element of the Lie algebra acting on a vector. With (1.16) as motivation, we define the map $\partial_{n}^{\natural}$ on the vector space

$$
V_{\mu}^{*} \otimes \bigwedge^{n}((\mathfrak{u} \oplus \overline{\mathfrak{u}}) \cap \mathfrak{k}) \otimes V_{Z}
$$

by

$$
\begin{aligned}
& \partial_{n}^{\natural}\left(v^{*} \otimes Y_{1} \wedge \cdots \wedge Y_{n} \otimes z\right) \\
& =\sum_{l=1}^{n}(-1)^{l}\left(\pi^{*}\left(Y_{l}\right) v^{*} \otimes Y_{1} \wedge \cdots \wedge \widehat{Y}_{l} \wedge \cdots \wedge Y_{n} \otimes z\right) \\
& \quad+(-1)^{l}\left(v^{*} \otimes Y_{1} \wedge \cdots \wedge \widehat{Y}_{l} \wedge \cdots \wedge Y_{n} \otimes Y_{l} z\right) .
\end{aligned}
$$

This map descends to a map that we shall also denote by $\partial_{n}^{\natural}$ on the space of $L$ invariants

$$
\left(V_{\mu}^{*} \otimes \bigwedge^{n}((\mathfrak{u} \oplus \overline{\mathfrak{u}}) \cap \mathfrak{k}) \otimes V_{Z}\right)^{L} .
$$

Because $\partial_{n}$ and $\partial_{n}^{\natural}$ both commute with the invariants functor, we see that producing an element of Ker $\partial_{n}$ for (1.15a) amounts to first producing an element of Ker $\partial_{n}^{\natural}$ 
for the space (1.17c) and then tensoring it with any element of $V_{\mu}$. Such is the procedure we follow when determining elements of Ker $\partial_{1}$ in Chapter 2. Here and elsewhere, when $n=1$ in $\partial_{n}$ or $\partial_{n}^{\natural}$, we shall drop the subscript 1 and simply write $\partial$ or $\partial^{\natural}$.

As a final note about calculating $\mathcal{L}_{S}(Z)$, in particular, the $(\mathfrak{k}, K)$ module that comes from the complex with modules $(1.14 \mathrm{~b})$, it is important to remember that, for $Y_{l} \in(\mathfrak{u} \oplus \overline{\mathfrak{u}}) \cap \mathfrak{k}$ and $T \in R(K)$,

$$
T Y_{l}=-r\left(Y_{l}\right) T
$$

We used this relation when computing the formula (1.16). It is also of particular importance when checking if a certain vector is in Ker $\partial_{S}$ or if a certain vector is in $\operatorname{Im} \partial_{S+1}$.

To determine a homology basis for each $K$ type that appears in $A_{\mathfrak{q}}(\lambda)$, we use the following definitions.

Definition 1.19. Suppose $(Z, \phi)$ is an irreducible, finite dimensional representation of $L, Z^{\#}$ being defined by (1.1), with $\phi$ extended in a natural way to a representation of $Z^{\#}$. Also, suppose $\left(V_{\mu}, \pi\right)$ and $\left(V_{\mu}{ }^{*}, \pi^{*}\right)$ are the representations mentioned in Definition 1.7. Then

$$
\left.\mathcal{C}_{n}^{*}(Z)\right|_{\mu}
$$

is the space of $L$ invariants

$$
\left(V_{\mu}^{*} \otimes \bigwedge^{n}((\mathfrak{u} \oplus \overline{\mathfrak{u}}) \cap \mathfrak{k}) \otimes U(\mathfrak{u}) \otimes Z^{\#}\right)^{L}
$$

for the representation $\pi^{*} \otimes \operatorname{Ad} \otimes \phi\left(L\right.$ acts trivially on $\left.\bigwedge^{n}((\mathfrak{u} \oplus \overline{\mathfrak{u}}) \cap \mathfrak{k})\right)$. This space is isomorphic to the vector space $(1.17 \mathrm{c})$, since $V_{Z} \cong U(\mathfrak{u}) \otimes Z^{\#}$, and is a functor from the category of finite dimensional $(\mathfrak{l}, L)$ modules to the category of complex vector spaces. When we wish to focus on the map Ker $\partial_{n}^{\natural}$ or the map $\operatorname{Im} \partial_{n+1}^{\natural}$ restricted to the $K$ type $\mu$ of a discrete series $\mathcal{L}_{S}(Z)$, we shall use the notation Ker $\left.\partial_{n}^{\natural} \subseteq \mathcal{C}_{n}^{*}(Z)\right|_{\mu}$ or $\left.\operatorname{Im} \partial_{n+1}^{\natural} \subseteq \mathcal{C}_{n}^{*}(Z)\right|_{\mu}$, respectively. Because $\operatorname{dim}\left(\left.\operatorname{Ker} \partial_{S}^{\natural} \subseteq \mathcal{C}_{S}^{*}(Z)\right|_{\mu} /\left.\operatorname{Im} \partial_{S+1}^{\natural} \subseteq \mathcal{C}_{S}^{*}(Z)\right|_{\mu}\right)$ is the multiplicity of a $K$ type in $\mathcal{L}_{S}(Z),\left(\left.\operatorname{Ker} \partial_{S}^{\natural} \subseteq \mathcal{C}_{S}^{*}(Z)\right|_{\mu} /\left.\operatorname{Im} \partial_{S+1}^{\natural} \subseteq \mathcal{C}_{S}^{*}(Z)\right|_{\mu}\right)$ is referred to as the multiplicity space of type $\mu$ for $\mathcal{L}_{S}(Z)$.

$$
\left.\mathcal{C}_{n, K}(Z)\right|_{\mu}
$$

is the space of $L$ invariants

$$
\left(V_{\mu} \otimes V_{\mu}^{*} \otimes \bigwedge^{n}((\mathfrak{u} \oplus \overline{\mathfrak{u}}) \cap \mathfrak{k}) \otimes U(\mathfrak{u}) \otimes Z^{\#}\right)^{L}
$$

for the representation $r \otimes \operatorname{Ad} \otimes \phi$, where $r=$ right regular representation on $R(K)$. This $(\mathfrak{k}, K)$ module is isomorphic to the module (1.15a) for the same reason as in (1.19a). Also, it is a functor from the category of finite-dimensional $(\mathfrak{l}, L)$ modules to the category of $(\mathfrak{k}, K)$ modules. Following the example in (1.19a), when we wish to focus on the map Ker $\partial_{n}$ restricted to the $K$ type $\mu$ of a discrete series $\mathcal{L}_{S}(Z)$, we shall use the notation $\left.\operatorname{Ker} \partial_{n} \subseteq \mathcal{C}_{n, K}(Z)\right|_{\mu}$. 
Once we produce a homology basis, we may reconstruct the full $\mathfrak{g}$ action. Note that reconstruction of the full $\mathfrak{g}$ action is really a reconstruction of the $\mathfrak{p}$ action, since $\left(\Pi_{\mathfrak{k}, L}^{\mathfrak{k}, K}\right)_{1}\left(\mathcal{F}_{\mathfrak{g}, L}^{\mathfrak{k}, L}\left(\operatorname{ind}_{\overline{\mathfrak{q}}, L}^{\mathfrak{g}, L}\left(Z^{\#}\right)\right)\right.$ is a $(\mathfrak{k}, K)$ module.

The key tools in reconstruction of the $\mathfrak{p}$ action are Propositions 1.20 and 1.21. Proposition 1.21 gives a $\mathfrak{p}$ action on homology by first lifting to cycles, then applying a map $\alpha_{1}$, and finally descending again to homology. The definition of $\alpha_{1}$ given in Proposition 1.20 is

$$
\alpha_{1}(X \otimes(T \otimes w))=T \otimes\left(\operatorname{Ad}(\cdot)^{-1} X\right) w
$$

where $X \in \mathfrak{g}, T \in R(K)$, and $w \in \bigwedge^{1}((\mathfrak{u} \oplus \overline{\mathfrak{u}}) \cap \mathfrak{k}) \otimes U(\mathfrak{u}) \otimes Z^{\#}$. Here, $Z^{\#}$ is the irreducible $\mathfrak{l}$ module defined in (1.1). If $\left\{X_{i}\right\}$ is an orthonormal basis of $\mathfrak{p}$, then by finite dimensional vector space theory,

$$
\operatorname{Ad}(\cdot)^{-1} X=\sum_{i}\left\langle\operatorname{Ad}(\cdot)^{-1} X, X_{i}\right\rangle X_{i}, \quad \text { for }\langle\cdot, \cdot\rangle=\text { inner product on } \mathfrak{g}
$$

and

$$
\begin{aligned}
T \otimes\left(\operatorname{Ad}(\cdot)^{-1} X\right) w & =\sum_{i} T \otimes\left\langle\operatorname{Ad}(\cdot)^{-1} X, X_{i}\right\rangle X_{i} w \\
& =\sum_{i}\left\langle\operatorname{Ad}(\cdot)^{-1} X, X_{i}\right\rangle T \otimes X_{i} w
\end{aligned}
$$

The second of these equalities follows from $[\mathrm{K}-\mathrm{V}, \mathrm{B} .18]$. This reference produces an isomorphism between a tensor product of spaces over $\mathbb{C}$ and a tensor product of spaces over another space of $C^{\infty}$ functions. Since $\left\langle\operatorname{Ad}(\cdot)^{-1} X, X_{i}\right\rangle$ is a $C^{\infty}$ function of $K$, we are able to pull it through the tensor product.

1.3. Useful theorems. In this section we present theorems that will be used in Chapter 2. We provide references after the statement of each theorem.

Proposition 1.20 (Duflo-Vergne). Let $(\mathfrak{g}, L) \hookrightarrow(\mathfrak{g}, K)$ be an inclusion of pairs, and let $V$ be in $\mathcal{C}(\mathfrak{g}, L)$. Regard $\bigwedge^{n} \mathfrak{c}$ as a trivial $(\mathfrak{g}, L)$ module, and make $\mathcal{F} V$ into a $(\mathfrak{g}, L)$ module so that its action is the same as in $V$. Let $\Phi_{0}$ be the Mackey isomorphism relative to $(\mathfrak{l}, L)$ and $(\mathfrak{k}, K)$. Then the $\mathcal{C}(\mathfrak{k}, K)$ map $\alpha_{n}$ that makes the diagram

$$
\begin{aligned}
& \mathfrak{g} \otimes_{\mathbb{C}}\left(R(K) \otimes_{L}\left(\bigwedge^{n} \mathfrak{c} \otimes_{\mathbb{C}} \mathcal{F} V\right)\right) \stackrel{\Phi_{0}^{-1}}{\longrightarrow} R(K) \otimes_{L}\left(\mathfrak{g} \otimes_{\mathbb{C}} \bigwedge^{n} \mathfrak{c} \otimes_{\mathbb{C}} \mathcal{F} V\right) \\
& R(K) \otimes_{L}(\bigwedge^{n} \mathfrak{c} \otimes_{\mathbb{C}} \overbrace{\mathcal{F} V))}^{\alpha_{n}}
\end{aligned}
$$

commute is given by

$$
\begin{array}{ll}
\alpha_{n}(X \otimes(T \otimes w))=T \otimes\left(A d(\cdot)^{-1} X\right) w & \text { for } X \in \mathfrak{g}, T \in R(K), \text { and } \\
& w \in \bigwedge^{n} \mathfrak{c} \otimes \mathcal{F} V .
\end{array}
$$

Proposition 1.21. Put

$$
V_{n}^{\Pi}=\bigwedge^{n} \mathfrak{c} \otimes_{\mathbb{C}} \mathcal{F} V \otimes_{\mathbb{C}}\left(\bigwedge^{m} \mathfrak{c}\right)^{*}
$$


Then the $\mathcal{C}(\mathfrak{k}, K)$ diagram

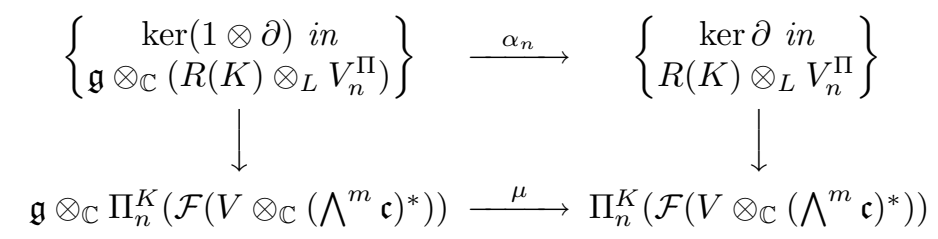

commutes.

Statements and proofs of these propositions may be found in $[\mathrm{K}-\mathrm{V}$, Propositions 3.80 and 3.83]. For our purposes, the important portion of Proposition 1.20 is the map $\alpha_{n}$. In Proposition 1.21, we may ignore the term $\left(\bigwedge^{m} \mathfrak{c}\right)^{*}$, since it is not relevant for our situation. Also, the functor $\Pi_{n}^{K}$ is the $n$th derived functor of $P_{\mathfrak{k}, L}^{\mathfrak{k}, K}$ (see $(1.9 \mathrm{~b})), \mathcal{F}=\mathcal{F}_{\mathfrak{g}, L}^{\mathfrak{k}, L}$ (see Definition 1.10), and the map $\mu$ is "multiplication by $\mathfrak{g}$ ", i.e., the $\mathfrak{g}$ action.

Proposition 1.22. In the case of $\mathrm{A}_{\mathfrak{q}}(\lambda)$, suppose that the inequality

$$
\operatorname{Re}\langle\lambda+\delta(\mathfrak{u}), \alpha\rangle \geq 0 \quad \text { for all } \alpha \in \Delta(\mathfrak{u})
$$

is satisfied. For $v \in \mathfrak{h}^{*}$, the space of linear functionals on the Cartan subalgebra $\mathfrak{h} \in \mathfrak{g}$, define $\mathcal{P}(v)$ to be the multiplicity of $v$ as a weight in $(S(\mathfrak{u} \cap \mathfrak{p}))^{\mathfrak{I} \cap \mathfrak{k} \cap \mathfrak{n}}$. Put $\Lambda=\lambda+2 \delta(\mathfrak{u} \cap \mathfrak{p})$, and let $W^{1}$ be the subset of $W(\mathfrak{k}, \mathfrak{h})$, the Weyl group of $K$, defined by

$$
W^{1}=\left\{w \in W(\mathfrak{k}, \mathfrak{h}) \mid \Delta^{+}(w) \subseteq \Delta(\mathfrak{u})\right\},
$$

where $\Delta^{+}(w)=\left\{\alpha \in \Delta^{+}(\mathfrak{k}) \mid w^{-1} \alpha<0\right\}$. If $\Lambda^{\prime}$ is $\Delta^{+}(\mathfrak{k})$ dominant and integral, then the $K$ type $\Lambda^{\prime}$ occurs in $\mathrm{A}_{\mathfrak{q}}(\lambda)$ with multiplicity

$$
\sum_{s \in W^{1}}(\operatorname{det} s) \mathcal{P}\left(s\left(\Lambda^{\prime}+\delta(\mathfrak{k})\right)-(\Lambda+\delta(\mathfrak{k}))\right) .
$$

The proof of this theorem may be found in $[\mathrm{K}-\mathrm{V}$, Theorem 8.29].

Cartan's Theorem. For each $\alpha \in \Delta(\mathfrak{g})$ a vector $X_{\alpha} \in \mathfrak{g}^{\alpha}$ can be chosen such that for all $\alpha, \beta \in \Delta(\mathfrak{g})$

$$
\begin{aligned}
& {\left[X_{\alpha}, X_{-\alpha}\right]=H_{\alpha}, \quad\left[H, X_{\alpha}\right]=\alpha(H) X_{\alpha} \quad \text { for } H \in \mathfrak{h} ;} \\
& {\left[X_{\alpha}, X_{\beta}\right]=0 \quad \text { if } \alpha+\beta \neq 0 \text { and } \alpha+\beta \notin \Delta(\mathfrak{g})} \\
& {\left[X_{\alpha}, X_{\beta}\right]=N_{\alpha, \beta} X_{\alpha+\beta} \quad \text { if } \alpha+\beta \in \Delta(\mathfrak{g})}
\end{aligned}
$$

where the constants $N_{\alpha, \beta}$ satisfy

$$
N_{\alpha, \beta}=-N_{-\alpha,-\beta}
$$

For any such choice

$$
N_{\alpha, \beta}^{2}=\frac{q(1-p)}{2} \alpha\left(H_{\alpha}\right),
$$

where $\beta+n \alpha(p \leq n \leq q)$ is the $\alpha$-series containing $\beta$.

For a proof, see [Hel, Theorem 5.5, p. 176]. In this theorem, $\mathfrak{g}^{\alpha}$ refers to the one-dimensional eigenspace of $\mathfrak{g}$ with eigenvalue $\alpha$ (see [Hel, p. 165] or [K3, p. 66]). 
Also, $H_{\alpha}$ is the unique vector in the Cartan subalgebra $\mathfrak{h}$ of $\mathfrak{g}$ that satisfies the relation

$$
B\left(H, H_{\alpha}\right)=\alpha(H) \quad \text { for all } H \in \mathfrak{h},
$$

where $B$ is the Killing form.

\section{2. $\mathfrak{g}$ ACTION FOR $\mathrm{A}_{\mathfrak{q}}(\lambda)$ MINIMAL $K$ TYPE}

In this chapter, we present some results that are true for a general quaternionic discrete series, defined in Chapter 1 . We will consider only the $A_{\mathfrak{q}}(\lambda)$ discrete series (Definition 1.13). In what follows, $\mathfrak{p}$ refers to the subspace of $\mathfrak{g}$ built from noncompact roots. Also, $\beta$ refers to the highest weight of $\mathfrak{k}$ (see Theorem 1.6(a)), and root vectors $X_{\alpha}$ are constructed by means of Cartan's Theorem (Chapter 1, Section 1.3). The term $\alpha_{0}$ refers to the largest noncompact root of $\mathfrak{g}$ (not to be confused with the map $\alpha_{n}$ in Propositions 1.20 and 1.21).

The first main result is a concrete realization of the multiplicity space of $\mathrm{A}_{\mathfrak{q}}(\lambda)$ for the minimal $K$ type $\lambda+2 \delta(\mathfrak{u} \cap \mathfrak{p})$, hereafter abbreviated by $\Lambda$, of $\mathrm{A}_{\mathfrak{q}}(\lambda)$. In fact, we shall show that $\left.\mathcal{C}_{2}^{*}\left(\mathbb{C}_{\lambda}\right)\right|_{\Lambda}$ is 0 , and as a result, the multiplicity space mentioned above will be Ker $\left.\partial^{\natural} \subseteq \mathcal{C}_{1}^{*}\left(\mathbb{C}_{\lambda}\right)\right|_{\Lambda}$. We precede this result with two lemmas; the first is used in the proof of the second, and the second will be used throughout this section.

Lemma 2.1. Suppose $G_{1}$ and $G_{2}$ are compact groups with $\pi_{1}, \pi_{2}$ irreducible representations of $G_{1}$ and $G_{2}$, respectively, on finite dimensional complex Hilbert spaces $V_{1}$ and $V_{2}$. Then we may assume $\pi_{1}$ and $\pi_{2}$ are unitary, by [K3, Proposition 1.6]. If the representation $\pi_{1} \otimes \pi_{2}$ of $G_{1} \times G_{2}$ on $V_{1} \otimes V_{2}$ is given by

$$
\left(\pi_{1} \otimes \pi_{2}\right)\left(g_{1}, g_{2}\right)=\pi_{1}\left(g_{1}\right) \otimes \pi_{2}\left(g_{2}\right) \quad \text { for } g_{1} \in G_{1} \text { and } g_{2} \in G_{2},
$$

then $\pi_{1} \otimes \pi_{2}$ is an irreducible representation. Conversely, if $\pi$ is an irreducible representation of $G_{1} \times G_{2}$, then $\pi \cong \pi_{1} \otimes \pi_{2}$, where $\pi_{1}$ is irreducible for $G_{1}$ and $\pi_{2}$ is irreducible for $G_{2}$.

Proof. To show the first statement, namely that $\pi_{1} \otimes \pi_{2}$ is an irreducible representation of $G_{1} \times G_{2}$, note that it is sufficient to show that, if

$$
\Phi: V_{1} \otimes V_{2} \rightarrow V_{1} \otimes V_{2}
$$

is a linear operator commuting with all $\left(\pi_{1} \otimes \pi_{2}\right)\left(g_{1}, g_{2}\right)$, then $\Phi$ is scalar. The reason for sufficiency is:

If $Z$ is an invariant subspace, then $Z^{\perp}$ is invariant since $\pi_{1} \otimes \pi_{2}$ is unitary and it follows that $V_{1} \otimes V_{2}=Z \oplus Z^{\perp}$. Let $\Phi$ be the identity on $Z$ and 0 on $Z^{\perp}$. One can check that $\Phi$ commutes with $\left(\pi_{1} \otimes \pi_{2}\right)\left(g_{1}, g_{2}\right)$.

Let us therefore assume that $\Phi$ is a linear operator satisfying the above condition. We wish to show that $\Phi$ is scalar. Let us take a basis of operators on $V_{1} \otimes V_{2}$ of the form $A_{i} \otimes B_{j} \in \operatorname{End}\left(V_{1}\right) \otimes \operatorname{End}\left(V_{2}\right)$. If $\Phi=\sum_{i, j} c_{i, j}\left(A_{i} \otimes B_{j}\right)$, we get the following 
identities by considering $g_{2}=1$ in $\left(\pi_{1} \otimes \pi_{2}\right)\left(g_{1}, g_{2}\right)$ :

$$
\begin{aligned}
& \Phi\left(\pi_{1}\left(g_{1}\right) \otimes I d\right)=\left(\pi_{1}\left(g_{1}\right) \otimes I d\right) \Phi, \\
& \sum_{i, j} c_{i, j}\left(A_{i} \otimes B_{j}\right)\left(\pi_{1}\left(g_{1}\right) \otimes I d\right)=\sum_{i, j} c_{i, j}\left(\pi_{1}\left(g_{1}\right) \otimes I d\right)\left(A_{i} \otimes B_{j}\right), \\
& \sum_{i, j} c_{i, j}\left(A_{i} \pi_{1}\left(g_{1}\right) \otimes B_{j}\right)=\sum_{i, j} c_{i, j}\left(\pi_{1}\left(g_{1}\right) A_{i} \otimes B_{j}\right) .
\end{aligned}
$$

Because $\left\{B_{j}\right\}$ is a basis for $\operatorname{End}\left(V_{2}\right)$, the last equation tells us that

$$
\sum_{i} c_{i, j} A_{i} \pi_{1}\left(g_{1}\right)=\sum_{i} c_{i, j} \pi_{1}\left(g_{1}\right) A_{i} \quad \text { for each } j .
$$

This equation says that for each $j, \sum_{i} c_{i, j} A_{i}$ commutes with $\pi_{1}\left(G_{1}\right)$. By a corollary of Schur's Lemma [K3, Corollary 1.9], it follows that $\sum_{i} c_{i, j} A_{i}=d_{j} I d$ for each $j$. Thus, we can write

$$
\begin{aligned}
\Phi=\sum_{j} \sum_{i} c_{i, j} A_{i} \otimes B_{j} & =\sum_{j} d_{j}\left(I d \otimes B_{j}\right) \\
& =I d \otimes \sum_{j} d_{j} B_{j} \\
& =I d \otimes B .
\end{aligned}
$$

We can repeat this argument with $\Phi$ and the linear operators $I d \otimes \pi_{2}\left(g_{2}\right)\left(g_{1}=\right.$ 1 in $\left.\pi_{1} \otimes \pi_{2}\left(g_{1}, g_{2}\right)\right)$ to conclude that $B$ is also scalar. From this we see that $\Phi$ is scalar and therefore $\pi_{1} \otimes \pi_{2}$ is irreducible.

Conversely, if $\pi$ is irreducible for $G_{1} \times G_{2}$, then the irreducible representations from the $\pi_{1} \otimes \pi_{2}$ construction above already give enough for the expansion of matrix coefficients.

Lemma 2.2. Suppose $K=S U(2) \times L_{s s}$, with $L_{s s}$ being the semisimple part of the compact group L. Let $\Lambda^{\prime}$ be $\Delta^{+}(\mathfrak{k})$ dominant and integral. If $V_{\Lambda^{\prime}}$ is an irreducible representation of $K$ with highest weight $\Lambda^{\prime}$, then the decomposition of $V_{\Lambda^{\prime}}$ into irreducible $L$ representations is given by the formula

$$
V_{\Lambda^{\prime}}=\sum_{k=0}^{2 M} V_{\Lambda^{\prime}-k \beta},
$$

with $M$ a nonnegative rational number such that $2 M \in \mathbb{Z}$.

Proof. For our situation of quaternionic discrete series, $L=S^{1} \times L_{s s}, S^{1} \subset S U(2)$ being the Cartan subgroup. From Lemma 2.1, it follows that an irreducible representation of $K$ with highest weight $\Lambda^{\prime}$ is of the form $V_{\Lambda_{1}} \otimes W_{\Lambda_{2}}$, where $V_{\Lambda_{1}}$ is an irreducible representation of $S U(2)$ with highest weight $\Lambda_{1}{ }^{\prime}, W_{\Lambda_{2}}{ }^{\prime}$ is analogously defined for $L_{s s}$, and $\Lambda_{1}{ }^{\prime}+\Lambda_{2}{ }^{\prime}=\Lambda^{\prime}$. Similarly, an irreducible representation of $L$ is of the form

(irreducible representation of $S^{1} \subset S U(2)$ )

$\times$ (irreducible representation of $\left.L_{s s}\right)$. 
If $\left\{v_{\Lambda_{1}{ }^{\prime}-k \beta}\right\}, 0 \leq k \leq \frac{2\left\langle\Lambda_{1}{ }^{\prime}, \beta\right\rangle}{|\beta|^{2}}$ is a basis of weight vectors for $V_{\Lambda_{1}{ }^{\prime}}$ (weights given by the subscripts), then each $v_{\Lambda_{1}{ }^{\prime}-k \beta}$ is a basis for the one-dimensional (therefore irreducible) representation $\mathbb{C} v_{\Lambda_{1}{ }^{\prime}-k \beta}$ of $S^{1} \subset S U(2)$. Again by Lemma 2.1, the set

$$
\mathbb{C} v_{\Lambda_{1}{ }^{\prime}-k \beta} \otimes W_{\Lambda_{2}{ }^{\prime}}, \quad 0 \leq k \leq \frac{2\left\langle\Lambda_{1}{ }^{\prime}, \beta\right\rangle}{|\beta|^{2}}
$$

is a collection of irreducible $L$ representations in $V_{\Lambda^{\prime}}$. If $M=\frac{\left\langle\Lambda_{1}{ }^{\prime}, \beta\right\rangle}{|\beta|^{2}}$, then clearly

$$
V_{\Lambda^{\prime}}=\sum_{k=0}^{2 M} \mathbb{C} v_{\Lambda_{1^{\prime}-k \beta}} \otimes W_{\Lambda_{2}{ }^{\prime}}
$$

Rewriting $\mathbb{C} v_{\Lambda_{1}{ }^{\prime}-k \beta} \otimes W_{\Lambda_{2}{ }^{\prime}}$ as $V_{\Lambda^{\prime}-k \beta}$ gives the statement of the lemma.

Throughout the remainder of this chapter, we shall use the notation $v$ to refer to vectors of $V_{\Lambda^{\prime}}$ or its contragredient.

Using the notation $V_{\Lambda} \otimes\left(V_{\Lambda}\right)^{*}$ as the $K$ isotypic subspace of $R(K)$ (see Definition 1.7), we have

Proposition 2.3. A basis vector for the multiplicity space of $\mathrm{A}_{\mathfrak{q}}(\lambda)$ for the minimal $K$ type $\Lambda$ is

$$
\left(v_{-\Lambda}\right)^{*} \otimes X_{-\beta} \otimes 1 \otimes 1,
$$

where $\left(v_{-\Lambda}\right)^{*}$ is a fixed vector in $\left(V_{\Lambda}\right)^{*}$ with weight given by its subscript.

Proof. As mentioned in the discussion before the statement of the proposition, we show that $\left.\mathcal{C}_{2}^{*}\left(\mathbb{C}_{\lambda}\right)\right|_{\Lambda}$ is 0 . Our first step in proving $\left.\mathcal{C}_{2}^{*}\left(\mathbb{C}_{\lambda}\right)\right|_{\Lambda}$ is 0 is to show that the weights of $\left(V_{\Lambda}\right)^{*}$ are

$$
\Lambda, \Lambda-\beta, \Lambda-2 \beta, \cdots,-\Lambda .
$$

We can show (2.3b) by showing that the weights of $V_{\Lambda}$ are those listed in (2.3b) and by noting that the weights of the contragredient representation $V^{*}$ are exactly the negatives of the weights of a representation $V$ (see [K2, p. 55]). The weight $\Lambda$ is a multiple of $\beta$, since the set of roots $\beta \cup$ \{simple roots of $l$ form a basis (over $\mathbb{R})$ for the space of all linear functionals of $\mathfrak{k}$, and $\Lambda \perp \Delta(\mathfrak{l})$ (from Definition 1.13). Also, because $\lambda$ is dominant and algebraically integral with respect to $\Delta^{+}(\mathfrak{k})$, it is a nonnegative integer multiple of $\beta$. Let us therefore write $\Lambda=M_{0} \beta$ for some nonnegative integer $M_{0}$. Humphreys shows [Hum, p. 125, Exercise 1], that if $\alpha \in \Delta(\mathfrak{k})$ and $0 \leq k \leq \frac{2\langle\Lambda, \alpha\rangle}{\langle\alpha, \alpha\rangle}$ for some symmetric, positive definite, bilinear form $\langle\cdot, \cdot\rangle$ on the Euclidean space of linear functionals of $\mathfrak{k}$, then

$$
m(\Lambda-k \alpha)=1,
$$

where $m(\alpha)$ refers to the multiplicity of the weight $\alpha$ in $V_{\Lambda}$. Using $\alpha=\beta$ and making the substitution $M_{0} \beta$ for $\Lambda$, we see that

$$
m\left(\left(M_{0}-k\right) \beta\right)=1 \quad \text { for } 0 \leq k \leq 2 M_{0} .
$$

This shows that the weights $(2.3 \mathrm{~b})$ are included in the set of weights of $V_{\Lambda}$. To see that these are the only weights, we note that $V_{\Lambda}$ decomposes as the direct sum $V_{\Lambda}=\bigoplus V_{\Lambda-k \beta}$ with $0 \leq k \leq 2 M_{0}$, by Lemma 2.2. Also, each weight $\Lambda-k \beta$ is orthogonal to $\Delta(\mathfrak{l})$ and hence $V_{\Lambda-k \beta}$ is a one-dimensional representation of $L$. 
This statement proves that the only weights of $V_{\Lambda}$ are in fact those listed in (2.3b). From the remarks above, the weights of $\left(V_{\Lambda}\right)^{*}$ are also those listed in $(2.3 \mathrm{~b})$. Let us fix nonzero vectors $v_{\Lambda-k \beta} \in V_{\Lambda-k \beta}$ and define linear functionals $\left(v_{-(\Lambda-k \beta)}\right)^{*}$ by $\left(v_{-(\Lambda-k \beta)}\right)^{*}=\left(\cdot, v_{\Lambda-k \beta}\right)$. Consider now the space

$$
\left(V_{\Lambda}\right)^{*} \otimes \bigwedge^{2}((\mathfrak{u} \oplus \overline{\mathfrak{u}}) \cap \mathfrak{k}) \otimes U(\mathfrak{u}) \otimes \mathbb{C}_{\Lambda+\beta},
$$

where we have used the equality $\Lambda+\beta=\lambda+2 \delta(\mathfrak{u})$. The space of $L$ invariants of $(2.3 \mathrm{~d})$ is $\left.\mathcal{C}_{2}^{*}(\mathbb{C})\right|_{\Lambda}$. The possible weights of a pure tensor in $(2.3 \mathrm{~d})$ are

$$
M_{0} \beta-k \beta+\sum_{\alpha \in \Delta(\mathfrak{u})} n_{\alpha} \alpha+\left(M_{0}+1\right) \beta,
$$

where $0 \leq k \leq 2 M_{0}$ and each $n_{\alpha}$ is a nonnegative integer. Since $\langle\beta, \alpha\rangle>0$ for every $\alpha \in \Delta(\mathfrak{u})$, then the inner product

$$
\left\langle\beta,\left(2 M_{0}+1-k\right) \beta+\sum_{\alpha \in \Delta(\mathfrak{u})} n_{\alpha} \alpha\right\rangle
$$

is always nonzero $\left(k \leq 2 M_{0}\right)$. Hence, any sum of weights $(2.3 \mathrm{e})$ is nonzero, and consequently, there are no nonzero tensors of total weight 0 in $(2.3 \mathrm{~d})$. We conclude that $\left.\mathcal{C}_{2}^{*}\left(\mathbb{C}_{\lambda}\right)\right|_{\Lambda}$ is 0 . From this it follows that Ker $\left.\partial^{\natural} \subseteq \mathcal{C}_{1}^{*}\left(\mathbb{C}_{\lambda}\right)\right|_{\Lambda}$ is actually an equality of sets. Notice that the possible weights of a pure tensor in

$$
\left(V_{\Lambda}\right)^{*} \otimes \bigwedge^{1}((\mathfrak{u} \oplus \overline{\mathfrak{u}}) \cap \mathfrak{k}) \otimes U(\mathfrak{u}) \otimes \mathbb{C}_{\Lambda+\beta}
$$

are

$$
M_{0} \beta-k \beta \pm \beta+\sum_{\alpha \in \Delta(\mathfrak{u})} n_{\alpha} \alpha+\left(M_{0}+1\right) \beta,
$$

where $k$ and $n_{\alpha}$ are as in (2.3e). Again, using the fact that $\langle\beta, \alpha\rangle>0$ for every $\alpha \in \Delta(\mathfrak{u})$, we see that a sum $(2.3 \mathrm{~g})$ will be 0 if and only if $k=2 M_{0}, n_{\alpha}=0$ for each $\alpha \in \Delta(\mathfrak{u})$, and we choose $-\beta$ from the set $\{ \pm \beta\}$. It follows that the only possible nonzero elements in $\left.\mathcal{C}_{1}^{*}\left(\mathbb{C}_{\lambda}\right)\right|_{\Lambda}$ are complex multiples of

$$
\left(v_{-\Lambda}\right)^{*} \otimes X_{-\beta} \otimes 1 \otimes 1,
$$

where $X_{-\beta} \in \Lambda^{1}((\mathfrak{u} \oplus \overline{\mathfrak{u}}) \cap \mathfrak{k})$ with weight $-\beta$. In order to complete our proof, we need only check that the tensor $(2.3 \mathrm{~h})$ is indeed $L$ invariant. From our discussion prior to $(2.3 \mathrm{~d})$, we know that $-\Lambda$ is an $L$ highest and an $L$ lowest weight in $\left(V_{\Lambda}\right)^{*}$. Therefore, by the Theorem of the Highest Weight, $X_{\varepsilon}\left(v_{-\Lambda}\right)^{*}=X_{-\varepsilon}\left(v_{-\Lambda}\right)^{*}=0$ for all $\varepsilon \in \Pi(\mathfrak{l})$. The same is true of the action of $X_{ \pm \varepsilon}$ on an element of $\mathbb{C}_{\Lambda+\beta}$. It follows that, for $\varepsilon \in \Delta(\mathfrak{l})$,

$$
\begin{aligned}
X_{\varepsilon}\left(\left(v_{-\Lambda}\right)^{*}\right. & \left.\otimes X_{-\beta} \otimes 1 \otimes 1\right) \\
& =\left(X_{\varepsilon}\left(v_{-\Lambda}\right)^{*}\right) \otimes X_{-\beta} \otimes 1 \otimes 1+\left(v_{-\Lambda}\right)^{*} \otimes X_{-\beta} \otimes X_{\varepsilon} \otimes 1 \\
& =0+\left(v_{-\Lambda}\right)^{*} \otimes X_{-\beta} \otimes 1 \otimes X_{\varepsilon} 1 \quad \text { since } \mathfrak{l} \subseteq \overline{\mathfrak{q}} \\
& =0+0 \\
& =0 .
\end{aligned}
$$

From this calculation we conclude that the vector $(2.3 \mathrm{~h})$ is $L$ invariant and therefore forms a basis for $\left.\mathcal{C}_{1}^{*}\left(\mathbb{C}_{\lambda}\right)\right|_{\Lambda}$, which in this case equals the multiplicity space of $\mathrm{A}_{\mathfrak{q}}(\lambda)$ for the minimal $K$ type $\Lambda$. This completes the proof. 
From Proposition 2.3 and Definition 1.19, we may write a basis for Ker $\partial \subseteq$ $\left.\mathcal{C}_{1, K}\left(\mathbb{C}_{\lambda}\right)\right|_{\Lambda}$ as

$$
\left.v_{\Lambda-k \beta} \otimes \operatorname{Ker} \partial^{\natural}\right|_{\Lambda} \quad \text { for } 0 \leq k \leq 2 M_{0} .
$$

For each $k, v_{\Lambda-k \beta}$ is a fixed nonzero basis element of $V_{\Lambda-k \beta}$, and $M_{0}$ is the nonnegative integer satisfying $\Lambda=M_{0} \beta$. The collection (2.4) is in fact a homology basis for the minimal $K$ type, because we showed in the proof of Proposition 2.3 that the space $\left.\mathcal{C}_{2}^{*}\left(\mathbb{C}_{\lambda}\right)\right|_{\Lambda}$ is 0 .

Our ultimate goal in this chapter is an explicit formula for the $\mathfrak{p}$ action on a basis vector in (2.4). To succeed, we must prove two results that are interesting in their own right. The first result gives a general form of any $K$ type appearing with nonzero multiplicity in $\mathrm{A}_{\mathfrak{q}}(\lambda)$, and the proof shows that there is only one nonidentity element in the set $W^{1}$ (defined in Proposition 1.22). The second result shows that $\left.\mathcal{C}_{0}^{*}\left(\mathbb{C}_{\lambda}\right)\right|_{\Lambda+\alpha_{0}}$ and $\left.\mathcal{C}_{2}^{*}\left(\mathbb{C}_{\lambda}\right)\right|_{\Lambda+\alpha_{0}}$ are 0 (recall $\alpha_{0}$ is the largest noncompact root in $\Delta)$. The proof of the second result uses characters and it places a restriction on those $K$ types $\mu$ in $\mathrm{A}_{\mathfrak{q}}(\lambda)$ for which $\left.\mathcal{C}_{0}^{*}\left(\mathbb{C}_{\lambda}\right)\right|_{\mu}$ and $\left.\mathcal{C}_{2}^{*}\left(\mathbb{C}_{\lambda}\right)\right|_{\mu}$ may be nonzero.

Proposition 2.5. The only nonidentity element of $W^{1}$ is $s_{\beta}$, which maps $\beta$ to $-\beta$ and leaves all other simple roots of $\mathfrak{k}$ fixed. Consequently, the only $\Delta^{+}(\mathfrak{k})$ linear functionals $\Lambda^{\prime}$ that appear with nonzero multiplicity in $\mathrm{A}_{\mathfrak{q}}(\lambda)$ are of the form

$$
\Lambda^{\prime}=\Lambda+\eta,
$$

where $\eta$ is an element of $S(\mathfrak{u} \cap \mathfrak{p})^{\mathfrak{I} \cap \mathfrak{n}}$. The multiplicity of $\Lambda^{\prime}$ is the multiplicity of $\eta$ in $S(\mathfrak{u} \cap \mathfrak{p})^{\mathfrak{l} \cap \mathfrak{n}}$.

Proof. We know from the definition of $W^{1}$ and from the fact that $\Delta(\mathfrak{u} \cap \mathfrak{k})=\beta$ that the only choices for $\Delta^{+}(w)$ (defined in Proposition 1.22) are the empty set $\varnothing$ and the one element set $\{\beta\}$. For an element $w$ in the Weyl group of $K$, Knapp and Vogan define $[\mathrm{K}-\mathrm{V},(4.133)]$ the length of $w$, denoted $l(w)$, by $l(w)=\left|\Delta^{+}(w)\right|$. We can easily verify that $l(w)=l\left(w^{-1}\right)$. Knapp shows [K3, pp. 80-81] that $l(w)$ is also the smallest number of factors needed to represent $w \in W$ as $w=s_{\alpha_{i_{k}}} \cdots s_{\alpha_{i_{1}}}$ with $\alpha_{i_{k}}, \cdots, \alpha_{i_{1}}$ in $\Pi(\mathfrak{k})$, a simple root system for $\mathfrak{k}$. Here, $s_{\alpha}$ is the root reflection defined by

$$
s_{\alpha}(\phi)=\phi-\frac{2\langle\phi, \alpha\rangle}{|\alpha|^{2}} \alpha \quad \text { for } \alpha \in \Delta \text { and } \phi \in\left(\mathfrak{h}_{0}\right)_{\mathbb{R}}^{\prime} .
$$

In this definition $\mathfrak{h}_{0}$ is a Cartan subalgebra of $\mathfrak{k}_{0},\left(\mathfrak{h}_{0}\right)_{\mathbb{R}}=i \mathfrak{h}_{0}$, and $\left(\mathfrak{h}_{0}\right)_{\mathbb{R}}^{\prime}$ denotes the (real) dual space of $\left(\mathfrak{h}_{0}\right)_{\mathbb{R}}$. Also, $\langle\cdot, \cdot\rangle$ in the definition of root reflection is some inner product defined on $\left(\mathfrak{h}_{0}\right)_{\mathbb{R}}^{\prime}$. For a further discussion, see $[K 3$, p. 69]. The definition of $s_{\beta}$ in the statement of the proposition follows from the definition of the root reflection and the fact that $\beta$ is orthogonal to all simple roots of $\mathfrak{l}$ with respect to the inner product mentioned in that definition.

Recall that the simple root system we choose for $\Delta(\mathfrak{k})$ is given by the set $\{\beta\} \cup$ $\Pi(\mathfrak{l})$. From the statement above, the only choice of lengths for $w \in W^{1}$ is either 0 or 1. If $l(w)=0$, then $w$ is the identity. If $l(w)=1$, then $w=s_{\alpha}$ for $\alpha \in \Pi(\mathfrak{k})$. Because $s_{\alpha}(\alpha)=-\alpha[\mathrm{K} 3$, Lemma 4.8] for each simple root $\alpha$, then $\alpha=\beta$ is the only choice that gives $\Delta^{+}\left(s_{\alpha}{ }^{-1}\right)=\{\beta\}$. Since $s_{\alpha}{ }^{-1}=s_{\alpha}$ for all roots $\alpha$, we conclude that $s_{\beta}$ is the only nonidentity element in $W^{1}$. 
To prove the second part of the proposition, we recall from Proposition 1.22 that the multiplicity of a $K$ type $\Lambda^{\prime}$ in $\mathrm{A}_{\mathfrak{q}}(\lambda)$ is given by

$$
\sum_{s \in W^{1}}(\operatorname{det} s) \mathcal{P}\left(s\left(\Lambda^{\prime}+\delta(\mathfrak{k})\right)-(\Lambda+\delta(\mathfrak{k}))\right),
$$

where $\mathcal{P}(v)$ is the multiplicity of $v$ as a weight in $(S(\mathfrak{u} \cap \mathfrak{p}))^{\mathfrak{r} \cap \mathfrak{n}}$. Let us consider the summand with $s=s_{\beta}$. We can rewrite this summand as

$$
\begin{aligned}
& s_{\beta}\left(\Lambda^{\prime}+\delta(\mathfrak{k})\right)-(\Lambda+\delta(\mathfrak{k})) \\
& \quad=s_{\beta}\left(\Lambda^{\prime}\right)+s_{\beta}(\delta(\mathfrak{k}))-\Lambda-\delta(\mathfrak{k}) \\
& \quad=\Lambda^{\prime}-\frac{2\left\langle\Lambda^{\prime}, \beta\right\rangle}{|\beta|^{2}} \beta+s_{\beta}(\delta(\mathfrak{k}))-\delta(\mathfrak{k})-\Lambda \quad \text { from the definition of } s_{\beta} .
\end{aligned}
$$

Knapp shows [K3, Proposition 4.33] that $s_{\beta}(\delta(\mathfrak{k}))=\delta(\mathfrak{k})-\beta$. Therefore, from $(2.5 \mathrm{~b})$, it follows that

$$
s_{\beta}\left(\Lambda^{\prime}+\delta(\mathfrak{k})\right)-(\Lambda+\delta(\mathfrak{k}))=\Lambda^{\prime}-\frac{2\left\langle\Lambda^{\prime}, \beta\right\rangle}{|\beta|^{2}} \beta-\Lambda-\beta .
$$

Because $\Lambda^{\prime}$ and $\Lambda$ are both $\Delta^{+}(\mathfrak{k})$ dominant, one can easily verify that the inner product of $(2.5 \mathrm{c})$ with $\beta$ is strictly negative. However, every weight that appears in $S(\mathfrak{u} \cap \mathfrak{p})$ has nonnegative inner product with $\beta$. Since the weights of $S(\mathfrak{u} \cap \mathfrak{p})^{\mathfrak{l} \cap \mathfrak{n}}$ are a subset of the weights of $S(\mathfrak{u} \cap \mathfrak{p})$, we see that the only contribution to the sum

$$
\sum_{s \in W^{1}}(\operatorname{det} s) \mathcal{P}\left(s\left(\Lambda^{\prime}+\delta(\mathfrak{k})\right)-(\Lambda+\delta(\mathfrak{k}))\right)
$$

comes from $s=1$, the identity. The second part of the proposition then follows immediately.

Proposition 2.6. $\left.\mathcal{C}_{0}^{*}\left(\mathbb{C}_{\lambda}\right)\right|_{\Lambda+\alpha_{0}}$ and $\left.\mathcal{C}_{2}^{*}\left(\mathbb{C}_{\lambda}\right)\right|_{\Lambda+\alpha_{0}}$ are 0 .

Proof. The first part of the proof will use a general $K$ type and only at the end of the proof will we consider the $K$ type $\Lambda+\alpha_{0}$. From Proposition 2.6, we know that

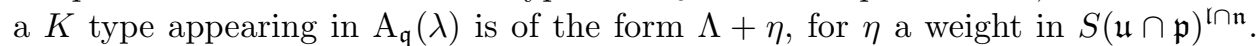
Consider the space

$$
\left.\left(V_{\Lambda+\eta}\right)^{*}\right|_{L} \otimes U(\mathfrak{u}) \otimes \mathbb{C}_{\Lambda+\beta}
$$

where $\left.\left(V_{\Lambda+\eta}\right)^{*}\right|_{L}$ is the representation $\left(V_{\Lambda+\eta}\right)^{*}$ of $K$ restricted to $L$. The space of $L$ invariants of $(2.6 \mathrm{a})$ is $\left.\mathcal{C}_{0}^{*}\left(\mathbb{C}_{\lambda}\right)\right|_{\Lambda+\eta}$. Because (2.6a) is an $L$ representation space and $L$ is compact, the Peter-Weyl Theorem says that (2.6a) breaks up as a direct sum of finite dimensional irreducible $L$ representations. The space $\left.\mathcal{C}_{0}^{*}\left(\mathbb{C}_{\lambda}\right)\right|_{\Lambda+\eta}$ is the subset of vectors in (2.6a) for which the action of $L$ is the trivial action. In other words, we have nontrivial $L$ invariance in (2.6a) if and only if the trivial representation of $L$ appears with nonzero multiplicity in the decomposition of (2.6a) into irreducible $L$ representations. Using Lemma 2.2 and the fact that the contragredient functor is additive $[\mathrm{K}-\mathrm{V}$, pp. 118 and 839] we see that

$$
\left(V_{\Lambda+\eta}\right)^{*}=\sum_{j=0}^{N}\left(V_{\Lambda+\eta-j \beta}\right)^{*}
$$


for some nonnegative integer $N$. Therefore, we can rewrite (2.6a) as

$$
\sum_{j=0}^{N}\left(V_{\Lambda+\eta-j \beta}\right)^{*} \otimes U(\mathfrak{u}) \otimes \mathbb{C}_{\Lambda+\beta} .
$$

Also, because $U(\mathfrak{u}) \otimes \mathbb{C}_{\Lambda+\beta}=\bigoplus_{n \in \mathbb{Z}^{+} \cup\{0\}} U^{n}(\mathfrak{u}) \otimes \mathbb{C}_{\Lambda+\beta}$ as $L$ representations, then we have reduced the problem to determining whether the trivial representation appears in

$$
\left(V_{\Lambda+\eta-j \beta}\right)^{*} \otimes U^{n}(\mathfrak{u}) \otimes \mathbb{C}_{\Lambda+\beta},
$$

for $0 \leq j \leq N$ (upper bound mentioned earlier) and any nonnegative integer $n$. This question is answered by using a result that appears in [Che] (Corollary 1, p. 188). We state a slightly modified version here:

The number of times that a finite dimensional irreducible representation $\tau_{1}$ of a compact Lie group $G$ appears in a finite dimensional representation $\tau$ is equal to

$$
\int_{G} \Theta_{\tau}(g) \overline{\Theta_{\tau_{1}}(g)} d g
$$

where $\Theta_{\tau}(\cdot)$ is the character function for the representation $\tau$ of $G$.

Chevalley [Che, p. 172] uses the term matrix representation $\tau(g)$ to mean a matrix realization of the endomorphism $\tau(g)$ with respect to a basis $v_{1}, \ldots, v_{n}$ of $V, \tau$ being a representation of $G$ on the finite dimensional Hilbert space $V$. He then defines [Che, Definition 1, p. 186] the character $\Theta_{\tau}(g)$ of $\tau(g)$ to be the trace of the matrix $\tau(g)$ with respect to the basis $v_{1}, \ldots, v_{n}$ of $V$. It is well known that the trace of $\tau(g)$ is independent of the choice of basis (see [H-K, Exercise 15, p. 106]). Some useful facts about characters are:

$$
\begin{array}{ll}
\Theta_{I d}(g)=1 & \\
\Theta_{\tau \otimes \phi}(g)=\Theta_{\tau}(g) \Theta_{\phi}(g) & \text { for representations } \tau \text { and } \phi \\
\Theta_{\left(\tau_{1}\right)^{*}}(g)=\overline{\Theta_{\tau_{1}}(g)} & \text { for irreducible unitary } \tau_{1} \text { and its } \\
& \text { contragredient }\left(\tau_{1}\right)^{*} .
\end{array}
$$

Returning now to the question of whether the trivial representation is in (2.6c), we note that Corollary 1 in [Che] and facts (1) and (3) about characters imply that this question is equivalent to determining whether or not the integral

$$
\int_{L} \Theta_{\left(V_{\Lambda+\eta-j \beta}\right)^{*} \otimes U^{n}(\mathfrak{u}) \otimes \mathbb{C}_{\Lambda+\beta}}(l) d l
$$

is nonzero. Using facts (2) and (3), we can rewrite (2.6e) as

$$
\int_{L} \overline{\Theta_{V_{\Lambda+\eta-j \beta}}(l)} \Theta_{U^{n}(\mathfrak{u}) \otimes \mathbb{C}_{\Lambda+\beta}}(l) d l .
$$

Since $V_{\Lambda+\eta-j \beta}$ is an irreducible representation of $L$, we can apply Corollary 1 again and conclude that the number of times that the trivial representation appears in $(2.6 c)$ is precisely number of times $\Lambda+\eta-j \beta$ can be written as $\gamma+\Lambda+\beta$, 
where $\gamma$ is an $L$ highest weight in the decomposition of $U^{n}(\mathfrak{u})$ into irreducible $L$ representations. Notice that because $\langle\beta, \alpha\rangle>0$ for every $\alpha \in \Delta(\mathfrak{u}), \gamma$ is also $\Delta^{+}(\mathfrak{k})$ dominant. We can rewrite the condition $(*)$ as number of times $\eta$ can be written as $\gamma+(j+1) \beta$,

with $j$ and $\gamma$ as above.

At this point, we specialize to the case where $\eta=\alpha_{0}$, the largest noncompact root. Because $j \geq 0$, we see that the left-hand side of

$$
\alpha_{0}-\beta-j \beta=\gamma,
$$

which is condition (2.6g) for the case $\eta=\alpha_{0}$, will not have positive inner product with $\beta$. Therefore, no choice of a $\Delta^{+}(\mathfrak{k})$ dominant $\gamma$ will give equality in $(2.6 \mathrm{~h})$. Notice that this conclusion is independent of the values $j$ and $n$; indeed, we only used that $j \geq 0$. Thus, we conclude that the trivial representation does not appear in

$$
\left.\left(V_{\Lambda+\alpha_{0}}\right)^{*}\right|_{L} \otimes U(\mathfrak{u}) \otimes \mathbb{C}_{\Lambda+\beta},
$$

and so $\left.\mathcal{C}_{0}^{*}\left(\mathbb{C}_{\lambda}\right)\right|_{\Lambda+\alpha_{0}}$ must be 0 . An analogous proof shows that $\left.\mathcal{C}_{2}^{*}\left(\mathbb{C}_{\lambda}\right)\right|_{\Lambda+\alpha_{0}}$ is 0 .

Having proven Propositions 2.5 and 2.6, we are ready to determine the action of a root vector $X \in \mathfrak{p}$ on a homology basis vector in (2.4).

Theorem 2.7. Let $\left.v_{\Lambda-k \beta} \otimes K e r \partial^{\natural}\right|_{\Lambda}$ be one of the basis vectors (2.4). If $X$ is a root vector in $\mathfrak{p}$, then we can realize the action

$$
X\left(\left.v_{\Lambda-k \beta} \otimes \operatorname{Ker} \partial^{\natural}\right|_{\Lambda}\right)=\alpha_{1}\left(\left.X \otimes v_{\Lambda-k \beta} \otimes \operatorname{Ker} \partial^{\natural}\right|_{\Lambda}\right)
$$

as an explicit formula. In fact, the only nonvanishing $K$ type in the decomposition of (2.7a) is $\Lambda+\alpha_{0}$.

Proof. Our goal is computing $\alpha_{1}\left(\left.X \otimes v_{\Lambda-k \beta} \otimes \operatorname{Ker} \partial^{\natural}\right|_{\Lambda}\right)$. We can rewrite this expression as

$$
\sum_{\alpha \in \Delta(\mathfrak{p})}\left\langle\left(\mathrm{Ad} \otimes \pi_{\Lambda}\right)(\cdot)^{-1} X \otimes v_{\Lambda-k \beta}, X_{\alpha} \otimes v_{\Lambda}\right\rangle \otimes X_{-\beta} \otimes X_{\alpha}(1 \otimes 1),
$$

and because $X_{\alpha}(1 \otimes 1)=0$ when $\alpha \in \Delta(\overline{\mathfrak{u}})$, we can rewrite $(2.7 \mathrm{~b})$ as

$$
\sum_{\alpha \in \Delta(\mathfrak{u} \cap \mathfrak{p})}\left\langle\left(\operatorname{Ad} \otimes \pi_{\Lambda}\right)(\cdot)^{-1} X \otimes v_{\Lambda-k \beta}, X_{\alpha} \otimes v_{\Lambda}\right\rangle \otimes X_{-\beta} \otimes X_{\alpha}(1 \otimes 1) .
$$

Of primary interest is the summand in (2.7c) for which $\alpha=\alpha_{0}$, the largest noncompact root of $\Delta(\mathfrak{g})$. We claim that $\alpha_{0}$ appears with multiplicity 1 in $S(\mathfrak{u} \cap \mathfrak{p})^{\mathfrak{n} \cap \mathfrak{n}}$. It certainly appears with multiplicity 1 in $S^{1}(\mathfrak{u} \cap \mathfrak{p})^{\mathfrak{l} \cap \mathfrak{n}}$. Because every positive noncompact root of $\mathfrak{g}$ is distinguished by the fact that it contains $\beta_{0}$ with coefficient 1 in its simple root expansion (recall $\beta_{0}$ is the unique simple root of $\Delta(\mathfrak{g})$ nonorthogonal to $\beta$ ), we see that $\alpha_{0}$ does not appear as a weight in $S^{N}(\mathfrak{u} \cap \mathfrak{p})$ for $N \geq 2$; consequently, it does not appear as a weight in $S^{N}(\mathfrak{u} \cap \mathfrak{p})^{\mathfrak{r} \cap \mathfrak{n}}$ and our claim is proved. We know from Proposition 2.5 that the $K$ type $\Lambda+\alpha_{0}$ appears with multiplicity 1 in $A_{\mathfrak{q}}(\lambda)$. Considering the space

$$
\left(V_{\Lambda+\alpha_{0}}\right)^{*} \otimes \bigwedge^{1}((\mathfrak{u} \oplus \overline{\mathfrak{u}}) \cap \mathfrak{k}) \otimes U(\mathfrak{u}) \otimes \mathbb{C}_{\Lambda+\beta},
$$


we next claim that the some nonzero multiple of the tensor

$$
\left(v_{-\left(\Lambda+\alpha_{0}\right)}\right)^{*} \otimes X_{-\beta} \otimes X_{\alpha_{0}} \otimes 1
$$

must appear as a summand of any nonzero vector in $\left.\mathcal{C}_{1}^{*}\left(\mathbb{C}_{\lambda}\right)\right|_{\Lambda+\alpha_{0}}$, which is the space of $L$ invariants of $(2.7 \mathrm{~d})$. In $(2.7 \mathrm{e}),\left(v_{-\left(\Lambda+\alpha_{0}\right)}\right)^{*}$ is some fixed nonzero vector in $\left(V_{\Lambda+\alpha_{0}}\right)^{*}$ with weight $-\left(\Lambda+\alpha_{0}\right)$. Proposition 2.6 shows that $\left.\mathcal{C}_{2}^{*}\left(\mathbb{C}_{\lambda}\right)\right|_{\Lambda+\alpha_{0}}=$ $\left.\mathcal{C}_{0}^{*}\left(\mathbb{C}_{\lambda}\right)\right|_{\Lambda+\alpha_{0}}=0$. This fact shows that a vector in (2.7d) is $L$ invariant if and only if it is in Ker $\partial^{\natural}$. To prove the claim about vector $(2.7 \mathrm{e})$, we note that a nonzero vector in $\left.\mathcal{C}_{1}^{*}\left(\mathbb{C}_{\lambda}\right)\right|_{\Lambda+\alpha_{0}}$ looks like

$$
\sum_{\omega, \gamma} c_{\omega, \gamma}^{-}\left(v_{\omega}\right)^{*} \otimes X_{-\beta} \otimes X_{\mathfrak{u}}^{\gamma} \otimes 1+\sum_{\omega, \gamma} c_{\omega, \gamma}^{+}\left(v_{\omega}\right)^{*} \otimes X_{\beta} \otimes X_{\mathfrak{u}}^{\gamma} \otimes 1 .
$$

In formula (2.7f), $\omega$ ranges over all the weights of $\left(V_{\Lambda+\alpha_{0}}\right)^{*}$. Also, if $\gamma_{1}, \ldots, \gamma_{n}$ is an ordering of the roots of $\mathfrak{u}$, then $\gamma$ is the tuple $\left(\gamma_{1}, \ldots, \gamma_{n}\right)$ and $X_{\mathfrak{u}}^{\gamma}$ is the monomial $X_{\gamma_{1}}^{r_{1}} \ldots X_{\gamma_{n}}^{r_{n}}$. Last, $c_{\omega, \gamma}^{-}$and $c_{\omega, \gamma}^{+}$are complex coefficients. We may assume that each of the tensor summands in (2.7f) is distinct. It is clear that any nonzero sum of tensors (2.7f) must contain weights $\omega$ that are $L$ lowest weights. From Lemma 2.2 and the definition of the contragredient representation, it follows that these weights are necessarily of the form $\omega=-\left(\Lambda+\alpha_{0}-k \beta\right.$ ), where $0 \leq k$ (the upper bound for $k$ is unimportant). Let us consider what pure tensors of total weight 0 in $(2.7 \mathrm{~d})$ have the form

$$
\left(v_{-\left(\Lambda+\alpha_{0}-k \beta\right)}\right)^{*} \otimes X_{-\beta} \otimes X_{\mathfrak{u}}^{\gamma} \otimes 1
$$

Or

$$
\left(v_{-\left(\Lambda+\alpha_{0}-k \beta\right)}\right)^{*} \otimes X_{\beta} \otimes X_{\mathfrak{u}}^{\gamma} \otimes 1 .
$$

In the case $(*)$, the monomial $X_{\mathfrak{u}}^{\gamma}$ is forced to have weight $\alpha_{0}-k \beta$. Because the weight of $X_{\mathfrak{u}}^{\gamma}$ must be $\Delta^{+}(\mathfrak{k})$ dominant, $k$ must be 0 . In the case $(* *)$, the monomial $X_{\mathfrak{u}}^{\gamma}$ is forced to have weight $\alpha_{0}-(k+2) \beta$. Since no choice of $k$ will make this weight be $\Delta^{+}(\mathfrak{k})$ dominant, our claim is proved. Notice that the only tensor in (2.7f) with term $\left(v_{-\left(\Lambda+\alpha_{0}\right)}\right)^{*}$ is $(2.7 \mathrm{e})$. This fact follows from the observation that the only elements of $U(\mathfrak{u})$ with weight $\alpha_{0}$ are multiples of $X_{\alpha_{0}}$. Since the multiplicity of $\Lambda+\alpha_{0}$ is 1 in $\mathrm{A}_{\mathfrak{q}}(\lambda)$, we know that the dimension of $\left.\mathcal{C}_{1}^{*}\left(\mathbb{C}_{\lambda}\right)\right|_{\Lambda+\alpha_{0}}$ is 1 (Definition 1.19 and Proposition 2.6). We define Ker $\left.\partial^{\natural}\right|_{\Lambda+\alpha_{0}}$ to be the nonzero element of $\left.\mathcal{C}_{1}^{*}\left(\mathbb{C}_{\lambda}\right)\right|_{\Lambda+\alpha_{0}}$ with summand (2.7e), i.e., the coefficient of the pure tensor $(2.7 \mathrm{e})$ is 1 in Ker $\left.\partial^{\natural}\right|_{\Lambda+\alpha_{0}}$. Having made this definition, we are now ready to determine the value of (2.7a). The expression $\alpha_{1}\left(\left.X \otimes v_{\Lambda-k \beta} \otimes \operatorname{Ker} \partial^{\natural}\right|_{\Lambda}\right)$ can be written

( $K$ type decomposition of $X \otimes v_{\Lambda-k \beta}$ )

$$
\times\left(K \text { type decomposition of } \alpha_{1}\left(\left.\cdot \otimes \operatorname{Ker} \partial^{\natural}\right|_{\Lambda+\alpha_{0}}\right)\right),
$$

where $\alpha_{1}\left(\left.\cdot \otimes \operatorname{Ker} \partial^{\natural}\right|_{\Lambda+\alpha_{0}}\right)$ is the expression gotten by replacing the term $(\operatorname{Ad} \otimes$ $\left.\pi_{\Lambda}\right)(\cdot)^{-1} X \otimes v_{\Lambda-k \beta}$ in $(2.7 \mathrm{c}$ ) with a $\operatorname{dot}$ (for an example with $G=S P(1,1)$, see [Gor], formula (3.22a)). Let us consider the $K$ type decomposition of $X \otimes v_{\Lambda-k \beta}$. We claim that, in the $K$ type decomposition of $\left.\mathrm{Ad}\right|_{\mathfrak{p}} \otimes \pi_{\Lambda}$, the only $K$ type that appears in the discrete series $\mathrm{A}_{\mathfrak{q}}(\lambda)$ is $\Lambda+\alpha_{0}$. To prove this claim, we note that the sum of two or more noncompact positive roots of $\mathfrak{g}$ cannot be a noncompact positive root. This fact follows from the characterization of noncompact positive roots as being 
those roots whose weight expansions in terms of simple roots have $\beta_{0}$ appearing exactly once. Next, we observe that any $K$ type appearing in $\left.\operatorname{Ad}\right|_{\mathfrak{p}} \otimes \pi_{\Lambda}$ is of the form $\Lambda+$ (noncompact root) ([K3, Exercise 13, p. 111]). Also, Proposition 2.5 says that any $K$ type appearing in $\mathrm{A}_{\mathfrak{q}}(\lambda)$ is of the form $\Lambda+\eta$, for $\eta$ an $L$ highest weight of $S(\mathfrak{u} \cap \mathfrak{p})^{\mathfrak{r} \cap \mathfrak{n}}$. Finally, we observe that $\beta$ is larger than any noncompact root. These four statements combined prove the claim. Notice this most recent claim proves the statement that the only nonvanishing $K$ type in the $K$ type decomposition of (2.7a) is $\Lambda+\alpha_{0}$. Using this information, we can write the $K$ type decomposition of $X \otimes v_{\Lambda-k \beta}$ as

$$
\begin{aligned}
& \left.h_{1} \text { (vector }_{1} \text { whose } K \text { type is } \Lambda+\alpha_{0}\right) \\
& +(\text { vectors whose } K \text { types do not appear in discrete series), }
\end{aligned}
$$

for some complex $h_{1}$. The only $K$ type of interest to us in the decomposition of $\alpha_{1}\left(\left.\cdot \otimes \operatorname{Ker} \partial^{\natural}\right|_{\Lambda}\right)$ is $\Lambda+\alpha_{0}$. In order to determine the portion with this $K$ type, we use the definition of Ker $\left.\partial^{\natural}\right|_{\Lambda+\alpha_{0}}$. It is clear from the formula (2.7c) and the definition of the expression $\alpha_{1}\left(\left.\cdot \otimes \operatorname{Ker} \partial^{\natural}\right|_{\Lambda}\right)$ that the sole contribution to the summand (2.7e) of Ker $\left.\partial^{\natural}\right|_{\Lambda+\alpha_{0}}$ comes from the term

$$
\left(\cdot, X_{\alpha_{0}} \otimes v_{\Lambda}\right) \otimes X_{-\beta} \otimes X_{\alpha_{0}} \otimes 1 .
$$

Under the $\left.\mathrm{Ad}\right|_{\mathfrak{p}} \otimes \pi_{\Lambda}$ decomposition, the tensor $X_{\alpha_{0}} \otimes v_{\Lambda}$ equals $\overline{h_{1}{ }^{\prime}} v_{\Lambda+\alpha_{0}}$ for some complex $h_{1}{ }^{\prime}$. There are no other terms appearing in the decomposition. Letting $\left(v_{-\left(\Lambda+\alpha_{0}\right)}\right)^{*}=\left(\cdot, v_{\Lambda+\alpha_{0}}\right)$, we have $\left\langle\cdot, X \otimes v_{\Lambda+\alpha_{0}}\right\rangle=h_{1}{ }^{\prime}\left(v_{-\Lambda+\alpha_{0}}\right)^{*}(\langle\cdot, \cdot\rangle$ is conjugatelinear in the second coordinate). Using (2.7i), we write the $K$ type decomposition of $\alpha_{1}\left(\left.\cdot \otimes \operatorname{Ker} \partial^{\natural}\right|_{\Lambda}\right)$ as

$$
\begin{aligned}
h_{1}{ }^{\prime} \operatorname{Ker} \partial^{\natural} & \left.\right|_{\Lambda+\alpha_{0}} \\
& +(\text { vectors whose } K \text { types do not appear in the discrete series). }
\end{aligned}
$$

From formulas $(2.7 \mathrm{~g}),(2.7 \mathrm{~h})$, and $(2.7 \mathrm{j})$ it follows that

$$
\alpha_{1}\left(\left.X \otimes v_{\Lambda-k \beta} \otimes \operatorname{Ker} \partial^{\natural}\right|_{\Lambda}\right)=h_{1} h_{1}{ }^{\prime} \text { vector }\left._{1} \otimes \operatorname{Ker} \partial^{\natural}\right|_{\Lambda+\alpha_{0}} .
$$

This completes the proof.

Remark. Formula (2.7c), which implicitly uses the fact that the $(\mathfrak{l}, L)$ module $\bigwedge^{1}((\mathfrak{u} \oplus \overline{\mathfrak{u}}) \cap \mathfrak{k})$ is a trivial module, shows that the coefficients $c_{\omega, \gamma}^{+}$in the sum (2.7f) are 0.

At this point, we continue with a discussion about the space $\left.\mathcal{C}_{2}^{*}\left(\mathbb{C}_{\lambda}\right)\right|_{\Lambda^{\prime}}$, with $\Lambda^{\prime}$ a $K$ type of nonzero multiplicity in $\mathrm{A}_{\mathfrak{q}}(\lambda)$. Gordon shows [Gor, Propositions 3.6 and 4.8] that $\left.\mathcal{C}_{2}^{*}\left(\mathbb{C}_{\lambda}\right)\right|_{\Lambda^{\prime}}$ is 0 for the case where $G=S P(1, n)$. The advantage for those groups is that, when computing a basis for homology, one need only consider $L$ invariance. We might ask whether this result is true for the general $\mathfrak{g}$. The answer unfortunately is no. We will show in the next proposition that, for any $K$ type $\Lambda^{\prime}$ that is a multiple of the largest root $\beta$, the space $\left.\mathcal{C}_{2}^{*}\left(\mathbb{C}_{\lambda}\right)\right|_{\Lambda^{\prime}}$ is nonzero. Because $\mathcal{L}_{2}\left(\mathbb{C}_{\lambda}\right)$ is $0\left[\mathrm{~K}-\mathrm{V}\right.$, Theorem 5.35], we see that Ker $\left.\partial_{2}^{\natural} \subseteq \mathcal{C}_{2}^{*}\left(\mathbb{C}_{\lambda}\right)\right|_{\Lambda^{\prime}}$ is 0 and hence any nonzero element in $\left.\mathcal{C}_{2}^{*}\left(\mathbb{C}_{\lambda}\right)\right|_{\Lambda^{\prime}}$ will map under $\partial^{\natural}$ to a nontrivial element in $\left.\operatorname{Im} \partial_{2}^{\natural} \subseteq \mathcal{C}_{1}^{*}\left(\mathbb{C}_{\lambda}\right)\right|_{\Lambda^{\prime}}$ 
Proposition 2.8. Suppose $\Lambda^{\prime}=M \beta$ is a $K$ type that appears in the discrete series $\mathrm{A}_{\mathfrak{q}}(\lambda)$ for $M \in \mathbb{Z}^{+}$satisfying $M \geq M_{0}+1\left(M_{0} \beta=\Lambda\right)$. Then $\left.\mathcal{C}_{2}^{*}\left(\mathbb{C}_{\lambda}\right)\right|_{M \beta}$ is a nontrivial vector space.

Remark. If $G=S O_{o}(4,4)$, then the Dynkin diagram corresponding to the simple roots of $\mathfrak{g}$ is $D_{4}$, and there are examples of $K$ types that are nonzero multiples of $\beta$ in this case.

Proof. $\left.\mathcal{C}_{2}^{*}\left(\mathbb{C}_{\lambda}\right)\right|_{M \beta}$ is the subset of $L$ invariant vectors of the space

$$
\left(V_{M \beta}\right)^{*} \otimes \bigwedge^{2}((\mathfrak{u} \oplus \overline{\mathfrak{u}}) \cap \mathfrak{k}) \otimes U(\mathfrak{u}) \otimes \mathbb{C}_{\Lambda+\beta}
$$

From formula $(2.3 \mathrm{~b})$ we know that the weights of $\left(V_{M \beta}\right)^{*}$ are

$$
M \beta,(M-1) \beta,(M-2) \beta, \cdots,-M \beta .
$$

Let $\left(v_{(M-k) \beta}\right)^{*}, 0 \leq k \leq 2 M$, be fixed nonzero elements of $\left(V_{M \beta}\right)^{*}$, with weights indicated by the subscripts. Consider the tensor (2.8a) given by

$$
\left(v_{-M \beta}\right)^{*} \otimes X_{-\beta} \wedge X_{\beta} \otimes X_{\beta}^{M-M_{0}-1} \otimes 1
$$

The claim is that this tensor is in fact $L$ invariant. To see this, notice first that $(2.8 \mathrm{~b})$ has weight 0 . Next, let $\varepsilon$ be any element of $\Pi(\mathfrak{l})$. Then $X_{\beta}$ and $X_{\varepsilon}$ commute in $U(\mathfrak{g})$ ( $\beta$ is the largest root) and $X_{\varepsilon}\left(v_{-M \beta}\right)^{*}=0$, since $\left(v_{-M \beta}\right)^{*}$ is an $L$ highest weight in $\left(V_{M \beta}\right)^{*}$ (proof of Proposition 2.3). It follows that

$$
\begin{aligned}
& X_{\varepsilon}\left(\left(v_{-M \beta}\right)^{*} \otimes X_{-\beta} \wedge X_{\beta} \otimes X_{\beta}^{M-M_{0}-1} \otimes 1\right) \\
& \quad=\left(X_{\varepsilon}\left(v_{-M \beta}\right)^{*}\right) \otimes X_{-\beta} \wedge X_{\beta} \otimes X_{\beta}^{M-M_{0}-1} \otimes 1 \\
& \quad \quad+\left(v_{-M \beta}\right)^{*} \otimes X_{-\beta} \wedge X_{\beta} \otimes X_{\varepsilon}\left(X_{\beta}^{M-M_{0}-1} \otimes 1\right) \\
& \quad=0+\left(v_{-M \beta}\right)^{*} \otimes X_{-\beta} \wedge X_{\beta} \otimes X_{\beta}^{M-M_{0}-1} \otimes X_{\varepsilon} 1 \\
& \quad=0,
\end{aligned}
$$

since $X_{\varepsilon} 1=0$. With $\varepsilon$ as above, we use the facts $(1) \mathfrak{k}$ is a Lie subalgebra of $\mathfrak{g}$ and (2) all the elements of $\Pi(\mathfrak{k})$ are linearly independent [K3, Proposition 4.6] when applying Cartan's Theorem to $\left[X_{\beta}, X_{-\varepsilon}\right]$ to conclude that the bracket is 0 , and hence $X_{\beta}$ and $X_{-\varepsilon}$ commute in $U(\mathfrak{g})$. We have $X_{-\varepsilon}\left(v_{-M \beta}\right)^{*}=0$ since $\left(v_{-M \beta}\right)^{*}$ is also an $L$ lowest weight, and so by replacing $X_{\varepsilon}$ by $X_{-\varepsilon}$ in (2.8c), we have that the tensor $(2.8 \mathrm{~b})$ is annihilated by $X_{-\varepsilon}$ for all simple roots $\varepsilon$ of $\mathfrak{l}$. This fact, in combination with the fact that vector $(2.8 \mathrm{~b})$ has weight 0 and is annihilated by $X_{\varepsilon}$ for all simple roots $\varepsilon$ of $\mathfrak{l}$, shows that vector $(2.8 \mathrm{~b})$ is an $L$ invariant tensor in the space (2.8a) and our result is proven.

Remark. Notice that we may repeat this argument with any pure tensor in (2.8a) that has total weight 0 if the term in $U(\mathfrak{u})$ is of the form $X_{\beta}^{n}$ for some $n \in \mathbb{Z}^{+} \cup\{0\}$, since each $\left(v_{(M-k) \beta}\right)^{*}$ is both an $L$ highest and $L$ lowest weight.

\section{ACKNOWLEDGEMENTS}

The author thanks Anthony W. Knapp, whose wisdom and guidance made this work a reality. The author also thanks his family for their unwavering love and support. 


\section{REFERENCES}

[Bar] V. Bargmann, Irreducible unitary representations for the Lorentz group, Ann. of Math. 48 (1947), 568-640. MR 9:133a

[Bes] A. Besse, Einstein Manifolds, Springer-Verlag, New York, 1987. MR 88f:53087

[BS-K] M. W. Baldoni-Silva and A. W. Knapp, Intertwining operators and unitary representations. I, J. Funct. Anal. 82 (1989), 151-236. MR 90g:22021

[Che] C. Chevalley, Theory of Lie Groups, Princeton University Press, Princeton, 1946. MR $7: 412 \mathrm{c}$

[Dix] J. Dixmier, Représentations intégrables du groupe de De Sitter, Bull. Soc. Math. France. 89 (1961), 9-41. MR 25:3384

[D-V] M. Duflo and M. Vergne, Sur le foncteur de Zuckerman, C. R. Acad. Sci. Paris Sér. I Math. 304 (1987), 467-469. MR 89h:22025

[EW1] T. J. Enright, R. Parthasarathy, N. R. Wallach, and J. A. Wolf, Classes of unitarizable derived functor modules, Proc. Nat. Acad. Sci. USA 80 (1983), 7047-7050.

[EW2] T. J. Enright, R. Parthasarathy, N. R. Wallach, and J. A. Wolf, Unitary derived functor modules with small spectrum, Acta Math. 154 (1985), 105-136. MR 86j:22026

[E-V] T. J. Enright and V. S. Varadarajan, On an infinitesimal characterization of the discrete series, Ann. of Math. 102 (1975), 1-15. MR 57:16472

[F1] R. Fueter, Die Funktionentheorie der Differentialgleichungen $\Delta u=0$ und $\Delta \Delta u=0$ mit vier reelen Variablen, Comment. Math. Helv. 7 (1935), 307-330.

[F2] R. Fueter, Über die analytische Darstellung der regulären Funktionen einer Quaternionenvariablen, Comment. Math. Helv. 8 (1936), 371-378.

[F3] R. Fueter, Die Singularitäten der eindeutigen regulären Funktionen einer Quaternionenvariablen, Comment. Math. Helv. 9 (1937), 320-335.

[FJ1] M. Flensted-Jensen, Spherical functions of a real semisimple Lie group. A method of reduction to the complex case, J. Funct. Anal. 30 (1978), 106-146. MR 80f:43022

[FJ2] M. Flensted-Jensen, Discrete series for semisimple symmetric spaces, Ann. of Math. 111 (1980), 253-311. MR 81h:22015

[Gor] D. Gordon, Quaternionic Discrete Series of Semisimple Lie Groups, Ph.D. Thesis, State University of New York, Stony Brook, 1995.

[HC1] Harish-Chandra, Representations of semisimple Lie groups. IV, Amer. J. Math. 77 (1955), 743-777. MR 17:282c

[HC2] Harish-Chandra, Representations of semisimple Lie groups. V, Amer. J. Math. 78 (1956), 1-41. MR 18:490c

[HC3] Harish-Chandra, Representations of semisimple Lie groups. VI, Amer. J. Math. 78 (1956), 564-628. MR 18:490d

[HC4] Harish-Chandra, Discrete series for semisimple Lie groups. II, Explicit determination of the characters, Acta Math. 116 (1966), 1-111. MR 36:2745

[Hel] S. Helgason, Differential Geometry, Lie Groups, and Symmetric Spaces, Academic Press, New York, 1978. MR 80k:53081

[H-K] K. Hoffman and R. Kunze, Linear Algebra, Second Edition, Prentice-Hall, Englewood Cliffs, N.J., 1971. MR 43:1998

[H-R] R. Hotta and R. Parthasarathy, Multiplicity formulae for discrete series, Invent. Math. 26 (1974), 133-178. MR 50:539

[Hum] J. E. Humphreys, Introduction to Lie Algebras and Representation Theory, SpringerVerlag, New York, 1972. MR 48:2197

[K1] A. W. Knapp, Introduction to Representations in Analytic Cohomology, Baston-Eastwood Summer Research Conference, 1992. MR 94k:22031

[K2] A. W. Knapp, Lie Groups, Lie Algebras, and Cohomology, Mathematical Notes No. 34, Princeton University Press, Princeton 1988. MR 89j:22034

[K3] A. W. Knapp, Representation Theory of Semisimple Groups: An Overview Based on Examples, Princeton University Press, Princeton, 1986. MR 87j:22022

[K4] A. W. Knapp, Bounded symmetric domains and holomorphic discrete series, Symmetric Spaces ( W.M. Boothby and G.L. Weiss, eds.), Marcel Dekker, New York, 1972, 211-246. MR 57:537

$[\mathrm{K}-\mathrm{V}]$ A. W. Knapp and D. Vogan, Cohomological Induction and Unitary Representations, Princeton University Press, Princeton, 1995. MR 96c:22023 
$[\mathrm{K}-\mathrm{W}]$ A. W. Knapp and N. R. Wallach, Szegö kernels associated with discrete series, Invent. Math., 34 (1976), 163-200. MR 82i:22016; MR 54:7704

[Lan] R. P. Langlands, Dimension of spaces of automorphic forms, Algebraic groups and Discontinuous Subgroups, Proc. Symp. in Pure Math. 9 (1966), American Mathematical Society, Providence, R.I., 235-257. MR 35:3010

[Rud] W. Rudin, Functional analysis, Second Edition, McGraw-Hill, New York, 1991. MR 92k:46001

[S1] W. Schmid, Homogeneous complex manifolds and representations of semi-simple Lie groups, Ph.D. dissertation, University of California, Berkeley, 1967, in "Representation Theory and Harmonic Analysis on Semisimple Lie Groups", Math. Surveys and Monographs, American Mathematical Society, Providence, (1989), 223-286. MR 90i:22025

[S2] W. Schmid, On the realization of the discrete series of a semisimple Lie group, Rice University Studies, 56 No. 2, (1970), 99-108. MR 43:3401

[S3] W. Schmid, $L^{2}$-cohomology and the discrete series, Ann. of Math., 103 (1976), 375-394. MR 53:716

[Sud] A. Sudbery, Quaternionic analysis, Math. Proc. Camb. Phil. Soc., 85 (1979), 199-225. MR 80g:30031

[Tak] R. Takahashi, Sur les représentations unitaires des groupes de Lorentz Généralisés, Bull. Soc. Math. France., 91 (1963), 289-433. MR 31:3544

[Vog] D. Vogan, Representations of Real Reductive Lie Groups, Birkhäuser, Boston, 1981. MR 83c: 22022

[Wol] J. Wolf, Complex homogeneous contact manifolds and quaternionic symmetric spaces, J. Math. and Mech. 14 (1965), 1033-1047. MR 32:3020

[Zuc] G. L. Zuckerman, Lecture Series, "Construction of representations via derived functors", Institute for Advanced Study, Princeton, N.J., Jan.-Mar. 1978.

Laboratory of Statistical Genetics, Rockefeller University, 1230 York Avenue, New YORK, NEW YORK 10021

E-mail address: gordon@morgan.rockefeller.edu 